\section{Expert Opinion}

1. Introduction

2. Background

3. Medical need

4. Existing treatment

5. Current research goals and scientific rationale

6. Competitive environment

7. Potential development issues

8. Expert opinion

\section{informa}

\section{healthcare}

\section{Emerging drugs for idiopathic pulmonary fibrosis}

\author{
Moisés Selman ${ }^{\dagger}$, Annie Pardo, Luca Richeldi \& Stefania Cerri \\ †Instituto Nacional de Enfermedades Respiratorias "Ismael Cosío Villegas", México
}

Introduction: Idiopathic pulmonary fibrosis (IPF) is a chronic, progressive and usually lethal lung disorder of unknown etiology. The disease is characterized by alveolar epithelial cell injury, formation of activated fibroblasts/ myofibroblasts foci and finally by the exaggerated accumulation of extracellular matrix with the subsequent destruction of the lung architecture. The long-term survival is distinctly poor, with only a $20-30 \%$ survival 5 years after the time of diagnosis. Actually, regardless of extensive research, no current therapy has been shown to either reverse or stop the progression of this disease.

Areas covered: The authors searched the Medline database from January 1990 to December 2010 using search terms 'pulmonary fibrosis', 'fibrosing alveolitis' and 'usual interstitial pneumonia'. Several subsets were included: definition and epidemiology, risk factors, clinical behavior, pathogenesis and therapy. For the section of IPF treatment, the authors examined all relevant studies including randomized controlled trials, cohort studies, casecontrol studies and cross-sectional studies. In this review, the authors describe the current therapeutic approaches, the ongoing clinical trials and some future options based on stem cells, lung bioengineering and microRNAs.

Expert opinion: The treatment of IPF represents one of the greatest challenges confronting respiratory medicine and, currently, there is no effective therapeutic option for IPF. Perhaps some of the drugs that are under evaluation in clinical trials will slow the decline of the pulmonary function tests or hopefully stabilize some patients. Nonetheless, it appears clear that new therapeutic approaches are urgently needed.

Keywords: IPF, lung bioengineering, microRNAs, stem cells, therapy

Expert Opin. Emerging Drugs [Early Online]

\section{Introduction}

Idiopathic pulmonary fibrosis (IPF) is a chronic, progressive and irreversible lung disorder of unknown etiology. The disease is characterized by alveolar epithelial cell injury and activation, followed by migration/proliferation and activation of mesenchymal cells with the formation of fibroblasts/myofibroblasts foci, and finally by the exaggerated accumulation of extracellular matrix with the subsequent destruction of the lung architecture. IPF is often (if not always) lethal, with a mortality rate that exceeds that of many cancers, and currently it lacks from any effective treatment. Importantly, IPF cannot be visualized as a simple scar, but rather a dynamic and complex biopathological process. However, the underpinning pathogenic mechanisms have not been elucidated, and only a detailed understanding of these mechanisms will open new and effective therapeutic strategies. Based on our current (although incomplete) understanding of the key pathogenic mechanisms and given the poor response of IPF patients to a variety of existing drugs, a growing number of clinical trials are going on aiming to target the cellular and molecular events believed to perpetuate the fibrotic response. 


\section{Background}

The exact incidence and prevalence of this disease are unknown, but it appears to be more common than previously supposed. Using a large, geographically diverse US healthcare claims database, Raghu et al. examined the prevalence and annual incidence of IPF [1]. By a narrow case-finding criteria, the prevalence ranged from 0.8 to $64.7 / 100,000$ individuals and the incidence from 0.4 to $7.1 / 100,000$ people [1]. In the UK, the incidence of IPF has increased progressively and has more than doubled between 1990 and 2003 [2]. The rising prevalence of IPF may be due to increased occurrence, recognition and diagnosis, or increased reporting. Both the prevalence and the incidence of IPF augment markedly with age, with prevalence exceeding 175 cases/100,000 individuals who are $>75$ years of age. Age also influences mortality and the median survival time is significantly shorter in older individuals compared with younger ones [3]. Actually, IPF is considered an aging related disorder although the mechanisms linking aging with the disease have not been elucidated [4]. Abnormal shortening of telomeres, oxidative stress and epigenetic modifications may contribute to this association [4].

Mortality appears also to be increasing. Using data from the National Center for Health Statistics (USA), Olson et al. found that the age-adjusted mortality rate for IPF increased $28.4 \%$ in men (from 40.2 deaths/1,000,000 in 1992 to 61.9 deaths $/ 1,000,000$ in 2003 ) and $41.3 \%$ in women (from 39.0 deaths $/ 1,000,000$ in 1992 to 55.1 deaths $/ 1,000,000$ in 2003) [5].

Although IPF appears to be inexorably progressive and lethal, the disease course is variable, with many patients remaining stable or progressing slowly for long periods of time while some others exhibit an accelerated course or experience acute exacerbations leading to respiratory failure and death [6-9]. In addition, examination of high resolution CT (HRCT) scan images has revealed that a number of patients with IPF, primarily smoker males, may display the combination of emphysematous lesions and pulmonary fibrosis, which has been called combined emphysema and pulmonary fibrosis [10,11]. These patients develop severe pulmonary hypertension and present decreased survival rate compared with patients with IPF alone [11,12]. Also, patients with IPF have higher risk to develop lung cancer. It is presently unknown whether this increase is related to the abnormal pathways upregulated in the disease or, as seems to be the case in IPF combined with emphysema, this relationship is not causal but directly associated to smoking [13].

\subsection{Diagnosis}

Specific diagnosis of IPF in patients having clinical and functional features of an interstitial lung disease relies on HRCT scanning or surgical lung biopsy showing a pattern of usual interstitial pneumonia (UIP). Characteristics findings of UIP by HRCT consist of bilateral, bibasilar and subpleural distribution of irregular interlobular septal thickening and honeycomb cysts often accompanied by traction bronchiectasis and minimal ground glass attenuation (Figure 1) [14]. The pathological pattern of UIP is characterized at low magnification by a remarkable temporal and geographic heterogeneous appearance with alternating zones of normal or nearly normal lung interspersed among widely scarred parenchyma (Figure 2) [15]. There is architectural distortion provoked by areas of honeycomb and fibrosis. Small clusters of spindleshaped fibroblasts and myofibroblasts within myxoid stroma (fibroblast foci) are present in the background of collagen deposition. These foci represent discrete, microscopic, subepithelial zones of ongoing fibrogenesis and are characteristic of UIP.

\subsection{Pathogenic mechanisms}

IPF is considered a complex disease probably arising from the interplay between genetic and environmental factors. Heterozygous mutations in surfactant protein (SP)-C and SFTPA2 genes, as well as in telomerase reverse transcriptase (TERT), and TERC (RNA that contains a template for telomere repeat addition) are probably responsible for about $15-20 \%$ of all familial interstitial pneumonias. In most families (80 - 85\%), vertical transmission suggesting single autosomal dominant mechanisms has been suggested, but the responsible genes have not been identified [16]. It is likely that different genes are responsible for disease across different families, contributing to difficulty in their discovery. On the other hand, a number of candidate genes have been evaluated in search for genetic factors in sporadic cases of IPF. Among others, polymorphisms of genes encoding for cytokines (e.g., IL-1- $\alpha$, TNF- $\alpha$ ), enzymes ( $\alpha 1$-antitrypsin, ACE, MMP-1), profibrotic molecules (TGF- $\beta$ ), and genes for SP-A and $-B$ have been studied [17]. Although some gene polymorphisms have been reported showing increased frequencies in patients with sporadic IPF, none of these findings has been replicated in subsequent studies. In addition, most of them have been performed in relatively small cohorts of patients. Therefore, association studies are inconclusive and at present there are no gene polymorphisms consistently associated with the sporadic form of the disease [17]. Genome-wide association studies that allow a genome-wide screen for risk factors of disease by a casecontrol association approach will probably open new windows to identify underlying genetic factors responsible for the development of sporadic IPF.

Several environmental exposures have been associated with increased risk for IPF. To date, cigarette smoking is the most consistent in both sporadic IPF and familial pulmonary fibrosis $[18,19]$. Also, several occupational factors, adjusted for age and smoking, have been found significantly associated with IPF, primarily metal and wood dust exposure $[20,21]$.

\subsection{Epithelial vs inflammation-driven fibrosis}

The pathogenesis of IPF is largely unknown. For a long time, the disease was considered as the result of a chronic unresolved inflammatory reaction of the lung that was 


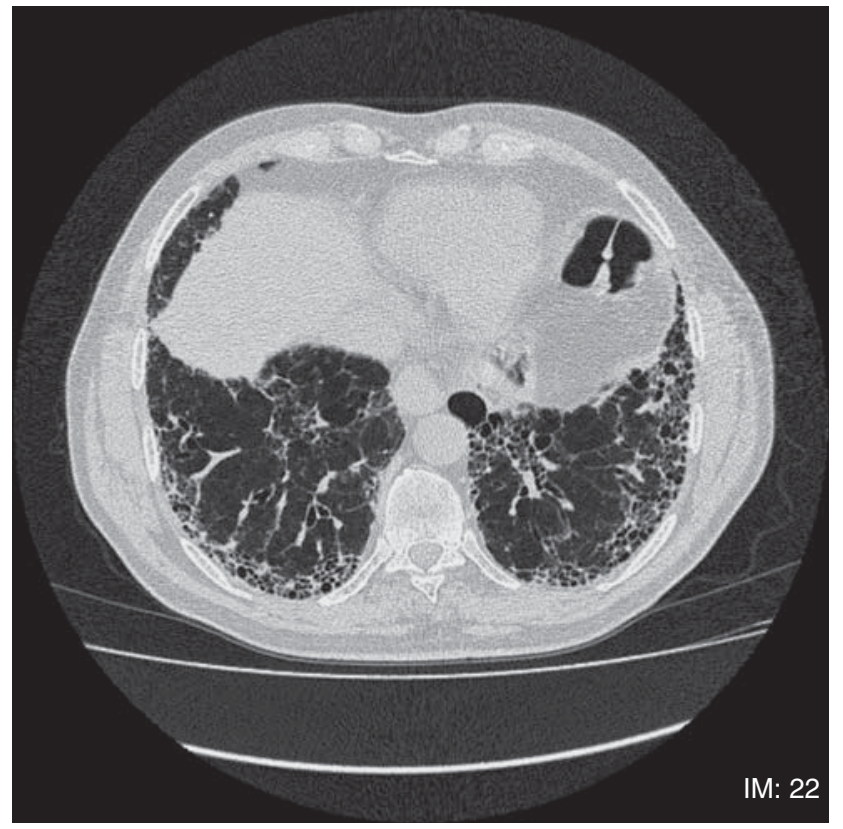

Figure 1. Usual interstitial pneumonia pattern on high resolution CT in an IPF patient. There are bilateral subpleural cysts in the lung bases (honeycombing), as well as reticulation and traction bronchiectasis.

IPF: Idiopathic pulmonary fibrosis.

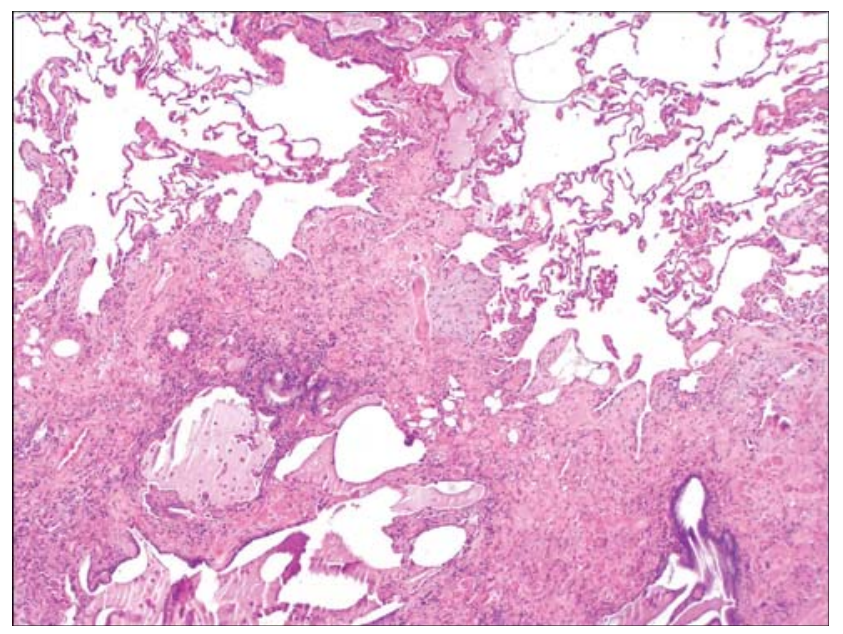

Figure 2. Usual interstitial pneumonia pattern on morphology. Low-magnification view illustrates the patchwork pattern of lung involvement with honeycombing alternating with parenchymal scarring and normal lung.

followed by fibroblast proliferation/activation and finally by the exaggerated accumulation of extracellular matrix proteins. However, in 2001 a new paradigm for pathogenesis, the epithelial-driven fibrosis, was proposed [22]. According to this hypothesis, alveolar epithelial cell microinjuries/apoptosis provoke the migration, proliferation and the aberrant activation of the neighboring epithelial cells, which in turn secrete the mediators responsible for the expansion of the fibroblasts/myofibroblasts population in the IPF lungs (Figure 3) [23]. This proposed model caused controversy, and several authors have debated that IPF is, as the other interstitial lung diseases, an inflammation-driven fibrosis [24]. In support of the inflammation as playing a role in IPF, there is some evidence suggesting that deregulated adaptive immune mechanisms and subsequent inflammation may be implicated either in the onset or in the progression of the disease in a subgroup of IPF patients [25]. Thus for instance, marked downregulation of CD28 on circulating CD4 T cells has been found in some patients with IPF and appears to identify patients at greatest risk for clinical deterioration [26]. Likewise, by gene microarray analysis, in addition to genes associated with tissue remodeling, an upregulation of genes related with chronic inflammation and immune response, such as cytokines, chemokines, complement and immunoglobulins, has been reported suggesting an active ongoing chronic inflammation [27]. More recently, several interleukins, that is, IL-17A, have been found to be increased in BAL fluids from IPF patients, and appeared to be critical for the development of experimental lung fibrosis [28]. Nevertheless, in a similar study IL-17R-deficient animals or specific IL-17A neutralization reduced the early inflammatory response but have no effect on the fibrotic response induced by silica [29]. However, a pathogenic role for inflammation cannot be excluded and repeated injury may superimpose persistent inflammation potentially enhancing disorderly tissue remodeling.

On the other hand, a growing body of evidence both in experimental models and in the human disease supports the epithelial hypothesis. First, several reports have demonstrated the presence of focal areas of alveolar epithelial apoptosis either in early or advanced stages of the disease [30-33] and, second, numerous investigations have found that in this disease, type II alveolar epithelial cells (AECIIs) synthesize virtually all the mediators responsible of the migration, proliferation and activation of the mesenchymal cells [23].

\subsection{Alveolar epithelial cell death}

The finding that SP-C mutations can cause epithelial cell death and familial IPF, highlights the importance of the AECIIs in the disease process [34,35]. The mutation results in an aberrant pro-SP-C protein that cannot be correctly processed, resulting in protein misfolding, accumulation, induction of endoplasmic reticulum stress (ERS) and apoptosis of the epithelial cells $[36,37]$. Recently, severe ERS response and associated apoptosis were revealed in the AECIIs of patients with sporadic IPF [38]. Furthermore, in another related work, ERS and unfolded protein response activation were found in AECIIs from patients with familial and sporadic IPF, but interestingly, expression of herpesvirus proteins was colocalized to this same cell population in the majority of patients [39]. This was an important finding because herpesviruses (primarily Epstein-Barr) are often found in 


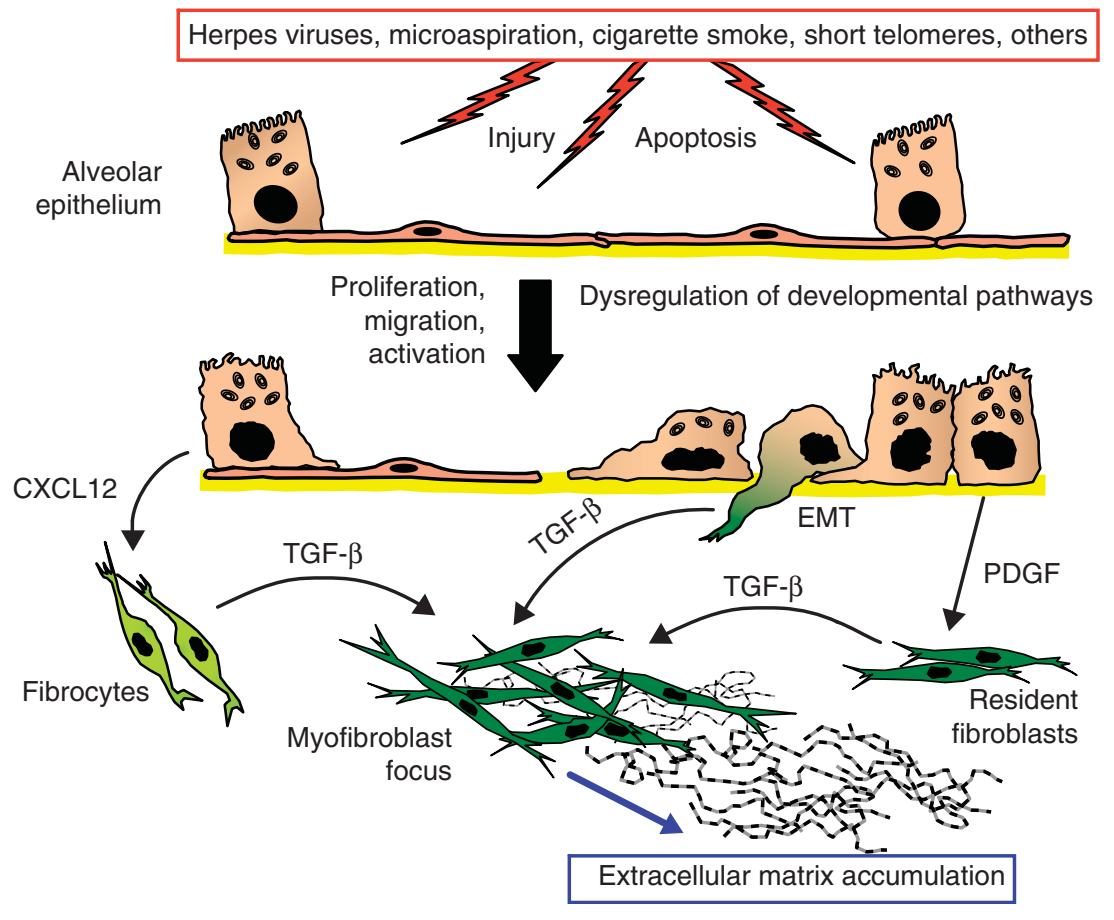

Figure 3. Schematic view of the main mechanisms involved in the pathogenesis of idiopathic pulmonary fibrosis. CXCL12: chemokine also known as stromal-derived factor-1; EMT: epithelial to mesenchymal transition; PDGF: platelet-derived growth factor.

the epithelial cells of IPF lungs, and they are one of the suspected risk factors for this disease [40]. Thus, viral replication may be one of the causes for the induction of ERS and repetitive injury to the alveolar epithelium in IPF.

Additional evidence in familial lung fibrosis also suggests that mutations resulting in epithelial cell injury and apoptosis may underlie the development of IPF. Heterozygous loss-offunction mutations in the human telomerase genes have been associated in close to $10 \%$ of familial IPF [41-43]. More recently, the relationship between telomerase and epithelial apoptosis was studied in sporadic IPF [44]. A significant inverse association was found between low AECIIs telomerase expression and apoptosis in apparent unaffected areas of IPF lungs. Electron microscopy confirmed epithelial apoptosis, alveolar collapse and initial fibroplasia. Taken together, these works indicate that abnormal AECIIs telomerase/apoptosis balance may reduce alveolar epithelial regenerative capacity, thus contributing to the early lung remodeling.

\subsection{Alveolar epithelial cell activation}

However, despite apoptosis, there is an increase in the number of alveolar/bronchiolar epithelial cells in the IPF lungs that appears to be aberrantly activated. Actually, different studies have demonstrated that they synthesized almost all, if not all, the growth factors responsible for the migration and proliferation of the resident lung fibroblasts and their differentiation to myofibroblasts (e.g., platelet-derived growth factor (PDGF), connective tissue growth factor (CTGF) and
TGF- $\beta 1$ ) [23]. In addition, alveolar epithelial cells may chemoattract bone marrow-derived circulating progenitors that also contribute to the expansion of the mesenchymal cells in the IPF lungs. For example, a recent work has demonstrated that in IPF, alveolar epithelial cells secrete CXCL12, a chemokine that participates in the trafficking/homing of circulating fibrocytes that express the receptor CXCR4 to the lungs [45]. Fibrocytes are a distinct subpopulation of leukocytes characterized by the simultaneous expression of mesenchymal (collagen type I, fibronectin), leukocyte (CD45) and hematopoietic stem cell (CD34) markers [46,47]. Importantly, they are precursors of myofibroblasts and may contribute to the expansion of lung myofibroblasts after lung injury. Fibrocytes express a variety of MMPs that may play a role in the process of fibrocyte migration throughout the basement membranes and the interstitial matrix, and may also be implicated in fibrotic tissue remodeling [48]. However, there is also some conflicting evidence for bone-marrow derivation of myofibroblasts. Thus, some studies performed primarily in mice indicate that bone marrow-derived cells are unlikely to give rise to $\alpha$-SMA-expressing progeny [49].

Finally, alveolar epithelial cells may contribute to the increase of lung fibroblasts/myofibroblasts through the epithelial to mesenchymal transition (EMT). Epithelial cell plasticity is a crucial and fascinating process in embryogenesis, characterized by the functional phenotypic transition of polarized epithelial cells into migratory, extracellular matrix producing mesenchymal cells. In IPF, several studies have 
revealed the presence of lung cells showing epithelial and mesenchymal markers, thus putatively transiting from alveolar epithelial cells to myofibroblasts $[50,51]$. TGF- $\beta$ is the main inducer of this process during development, carcinogenesis and tissue fibrosis with different isoforms mediating various effects depending on specific cellular context [52]. Interestingly, viral infection seems to potentiate the effect of TGF- $\beta$. In a recent work, it was shown that the latent membrane protein 1 (LMP-1) from the Epstein-Barr virus sensitizes alveolar epithelial cells to TGF- $\beta 1$-induced EMT response. Thus, at concentrations of TGF- $\beta 1$ below the threshold to cause EMT, LMP-1 induced dramatic phenotypic changes with concurrent loss of epithelial markers and increased cellular motility [53]. However, the magnitude of the contribution of EMT in tissue fibrosis is still under some debate. Thus, for example, a recent study in kidney fibrosis suggested that epithelial cells do not directly contribute to interstitial myofibroblast cells in vivo [54]. By using either red fluorescent protein or $\beta$-galactosidase as fate markers, no evidence that epithelial cells migrate outside of the tubular basement membrane and differentiate into interstitial myofibroblasts was found. Moreover, this study shows that pericyte differentiation accounted for a large majority of myofibroblasts at least in this model.

In a nutshell, strong evidence indicate that in IPF, activated alveolar epithelial cells provoke the expansion of the fibroblasts and myofibroblasts that cluster in the fibroblastic foci through the secretion of growth factors and chemokines that induce the migration, proliferation and activation of local lung fibroblasts as well as circulating progenitors, and also directly transiting to myofibroblasts by the EMT process.

\subsection{Alveolar epithelial cells and extracellular proteases}

In IPF, AECIIs also express several MMPs, for example, MMP-1 and -7, although their function in this disease is still unclear. These enzymes may be involved in extracellular matrix remodeling, basement membrane disruption and/or epithelial cell migration [55].

During the early stages of epithelial injury, a provisional matrix composed of fibronectin and fibrinogen is formed, and in order to facilitate the controlled alveolar re-epithelialization this matrix should be degraded [56]. In this context, decreased alveolar fibrinolysis provoking a local hypercoagulable state appears to be implicated in the pathogenesis of IPF. Importantly, strong evidence supports that AECIIs are mainly responsible of this pathological process. Thus, it has been demonstrated in IPF that tissue factor, FVII, and FX are synthesized by AECIIs and colocalize in the alveolar epithelium overlying fibroblast foci, probably allowing the activation of FX to FXa that in turn influences fibroblast functions, that is, enhancing fibroblast-to-myofibroblast differentiation, within the underlying fibrotic regions [57].

The molecular mechanisms responsible of the aberrant behavior of the alveolar epithelial cells in IPF are unknown, but recent evidence indicates that some developmental signaling pathways are aberrantly activated or dysregulated [58]. Thus, for example, Wnt/ $\beta$-catenin pathway is strongly upregulated in the IPF lung epithelium and the Wnt1-inducible signaling protein-1 (WISP1) is also increased in the AECIIs of these patients [59,60]. Treatment of AECIIs with recombinant WISP1 increases proliferation and induces EMT [60]. Abnormal recapitulation of embryologic pathways may also affect fibroblast behavior. Thus, gremlin, an antagonist of the bone morphogenetic protein (BMP)-4 during lung development, is overexpressed in IPF lungs, primarily in fibroblasts [61]. Moreover, TGF- $\beta$-induced EMT of lung epithelial cells in culture is associated with induction of gremlin mRNA expression [61].

\section{Medical need}

IPF patients have been treated for a long time with corticosteroids and in the last 15 years with a combination of corticosteroids and immunosuppressive drugs [62]; however, a systematic appraisal of the level of evidence available for these treatments $[63,64]$ showed that insufficient data support this approach. Furthermore, although in the absence of a formal demonstration of a lack of efficacy, clinical experience confirms in daily practice that corticosteroids do not affect the natural history of this disease.

Over the last years, despite the impressive increase in the number of published studies on IPF pathogenesis (from 68 listed in PubMed for the first 10 months of 2000 to 245 listed for the same period of time in 2010), no useful drug has reached most patients yet. As such, and in light of the many practical and psychological obstacles posed by lung transplantation, the medical need in IPF is one of the highest among people affected with chronic respiratory disorders.

Overall, there is a consensus within the international medical community that high-quality, prospective, controlled, clinical trials of new therapies for IPF are urgently required, although it is widely recognized that an effective treatment for such a complex disease will require, analogous with cancer, a combination of therapies targeting different multiple molecular and cellular pathways. Additionally, there is a need for targeting in future clinical trials end points of proven clinical value, also acceptable to different regulatory agencies. As of today, such clinical end points have been only partially identified and agreed upon that overall mortality does not appear a feasible and realistic target for future trials. The end points of such future studies will need to be chosen based on different characteristics of various study populations, such as the extent of disease and the presence of co-morbidities.

Finally, future studies and collaborative networks will require addressing issues on non-pharmacological management of IPF patients, including physical rehabilitation, psychological aspects and end-of-life procedures. It is unfortunate that in many institutions, terminally ill patients with IPF are not managed accordingly to best care procedures, shared 
among different centers and focused on assuring adequate comprehensive support to patients and their families.

\section{Existing treatment}

The evolving hypotheses on pathogenesis of IPF have changed also varying over time the therapeutic approach to this devastating disease. As mentioned, the classical view considered IPF as a disease in which lung fibrosis was the consequence of a chronic inflammation initiated by an unidentified insult and ultimately leading to scarring. As such, corticosteroids have been the initial mainstay of treatment, in association with other immunosuppressive drugs, such as azathioprine. However, newly emerging concepts began to highlight the fact that IPF is likely to originate as a consequence of an abnormal wound healing process in which inflammation does not play a central role. The injury to the alveolar epithelial cell layer, through the secretion of several profibrotic mediators, is associated with the formation of patchy fibroblast-myofibroblast foci and deposition of extracellular matrix: in this scenario, there is little rationale for any anti-inflammatory treatment. As a consequence of this change of perspective on the pathogenesis of IPF, over the last decade a number of basic research studies led to translational studies exploring innovative therapeutic approaches, moving from an anti-inflammatory profile to a more anti-fibrotic mechanism of action.

\subsection{Corticosteroids}

Corticosteroids are synthetic molecules derived from the chemical structure of cortisol, the most important human glucocorticoid hormone. These compounds have several biological effects, which can be grouped into two broad categories, metabolic (particularly on glucose metabolism) and immunological effects. The action of corticosteroids on the immune system results in suppression of both cellular and humoral immune response and in a reduction on the levels of pro-inflammatory molecules.

Results of some clinical trials exploring the efficacy of corticosteroids in the treatment of adults with IPF are summarized in a recently updated Cochrane review [63]. Compared to the previous version of the review published in 2003, the current update further highlights the fact that in recent years there has been a lack of interest in the use of corticosteroid therapy alone in the treatment of IPF, as there was no new clinical trial on this topic eligible for inclusion in a systematic review and meta-analysis. Based on the results of this review and on the current understanding of the pathogenesis of $\mathrm{IPF}$, there is no reasonable evidence supporting the use of corticosteroids in this disease.

\subsection{Azathioprine}

Azathioprine is an anti-metabolite compound, orally available and well absorbed by the gastrointestinal tract. The drug blocks most T-cell functions, inhibits primary antibody synthesis, and decreases the number of circulating monocytes and granulocytes. Due to its immunomodulatory properties, azathioprine was thought to be suitable for the treatment of IPF, mostly given in association with corticosteroid therapy, as recommended in the ATS/ERS Guidelines published in 2000 [62]. Only one randomized clinical trial on the efficacy of azathioprine in IPF has been published [65]. The trial was a prospective, double-blind, randomized, placebocontrolled study comparing the therapeutic effect of combined prednisone/azathioprine with that of prednisone/ placebo in a very small group of IPF patients. Azathioprine showed a small benefit on survival only when the analysis was adjusted for age as a continuous variable at up to 9 years of follow-up. With respect to the change in lung function from baseline, although trends to improvement with azathioprine were observed, the difference between the treatment and the control groups was not statistically significant.

\section{3 $\mathrm{N}$-acetylcysteine}

$\mathrm{N}$-acetylcysteine (NAC) acts as a precursor of the major antioxidant glutathione. The rationale for the use of NAC in the treatment of IPF is based on findings demonstrating that there is an oxidant-antioxidant imbalance in this disease. In addition, high-dose NAC has shown some immunemodulatory properties in a different lung disease such as cystic fibrosis [66]. Given its properties, NAC was evaluated in a randomized clinical trial for the treatment of IPF in which patients received either high-dose NAC or placebo in combination with prednisone and azathioprine [67]. While survival was not affected by the treatment, the addition of NAC showed a marginal positive effect in the change of DLCO from baseline. NAC also was shown to slow the deterioration of forced vital capacity (FVC) at 12 months, although the difference in the absolute change of FVC from baseline between the treatment and the control arm failed to reach a conventional statistical significance. A subsequent analysis of the data of patients who completed this trial showed that lung function indices did not significantly change in patients receiving NAC while deteriorated in those included in the placebo arm [68]. Due to the lack of a true placebo arm in this trial, it is difficult to argue whether the observed effect is attributable only to the efficacy of NAC or is the result of the interaction between drugs with different mechanisms of action. Therefore, the National Institute of Health (NIH) is currently sponsoring a new randomized clinical trial assessing the efficacy of NAC alone compared to placebo.

\subsection{Pirfenidone}

Pirfenidone is an orally available small, synthetic non-peptide molecule that is easily absorbed from the gastrointestinal tract and that exhibits antifibrotic, anti-inflammatory and antioxidant properties in several models. Pirfenidone suppresses lung inflammation and fibrosis via inhibition of TGF- $\beta$ induced collagen synthesis, inhibition of TNF- $\alpha$ production and inhibition of the p-38 $\gamma$ MAPK [69-72]. Promising results from an open-label Phase II study supported the use of this 
drug in the treatment of IPF [73]. Subsequently, two randomized clinical trials assessing the efficacy of pirfenidone compared to placebo in IPF patients have been already published [74,75]. Two additional large multi-center randomized clinical trials have been completed and data have been publicly released to the FDA [76]. All four randomized clinical trials have been included in a recently published Cochrane review on non-steroid agents for IPF [64]. Based on the meta-analyses presented in this systematic review, pirfenidone appears to reduce the risk of disease progression by $30 \%$ and provide a beneficial effect on the change of VC from baseline, compared to placebo. Some limitations to this interpretation still apply, mostly related to a certain degree of methodological heterogeneity between studies (i.e., the methods for reporting lung function results), which did not allow including the results of all four trials in one meta-analysis. However, these data look encouraging and when considering the cumulative effect on progression-free survival and the relatively safe profile of this drug, pirfenidone appears a reasonable choice to treat IPF. At present, pirfenidone has been already approved for clinical use in IPF patients in Japan and India, and more recently it received also the approval by the European Medicines Agency for its use in Europe, while it has not been approved in the US.

\subsection{IFN- $\gamma$}

IFN- $\gamma$ is an endogenously cytokine with antifibrotic, antiinfective, antiproliferative and immunomodulatory properties. In several experimental models, IFN- $\gamma$ exhibited a dose-dependent inhibition of fibroblast proliferation, collagen-matrix deposition and collagen synthesis [77-79]. In addition, exogenous IFN- $\gamma$ was shown to downregulate the transcription of the TGF- $\beta$ gene in a model of bleomycininduced fibrosis [80]. On these bases, IFN- $\gamma$ was thought to be a potential drug for the treatment of IPF. First data on the use of IFN- $\gamma 1 \mathrm{~b}$ in IPF patients appeared in 1999 by Ziesche et al. [81] and showed a significant beneficial effect of this molecule in a very small single-center study. Two large placebo-controlled randomized clinical trials were then performed assessing the efficacy of IFN- $\gamma$ 1b in IPF patients [82,83]. However, the results of these trials did not confirm originally observations from Ziesche et al. Actually, as demonstrated in recent meta-analyses, this drug does not appear to be more effective than placebo neither on survival nor on the change of lung function from baseline. Given these data, there is no evidence to support any further use of IFN- $\gamma$ $1 \mathrm{~b}$ alone in the treatment of IPF.

\subsection{Imatinib}

Imatinib mesilate is an orally available signal transduction inhibitor, originally developed as an anticancer and approved for the treatment of chronic myelogenous leukemia. Imatinib is a specific tyrosine kinase inhibitor, with activity against Bcr-Abl, PDGF receptors (PDGFR) and c-kit [84,85]. The inhibitory activity on PDGFR suggested a potential activity of the drug in IPF, through the suppression of the profibrotic and proliferative activities mediated by PDGF. In fact, in preclinical models imatinib was shown to be able to inhibit bleomycin-induced lung fibrosis [86]. Daniels et al. have recently published the results of a randomized clinical trial on imatinib in patients with IPF [87]. This trial failed to demonstrate a benefit for imatinib compared to placebo on any of the outcomes selected for this study, and in particular did not show a statistically significant difference between the treatment and the control group in the progression-free survival index or in the change of lung function from baseline.

\subsection{Bosentan and other endothelin receptor antagonists}

Bosentan is an oral sulfonamide-based endothelin- $A$ and -B receptor antagonist, which has been developed for the treatment of pulmonary arterial hypertension. Endothelin-1 is an endogenous vasoconstrictor, which is involved in the pathogenesis of pulmonary arterial hypertension. In addition to the effects on vascular remodeling and vasoconstriction, endothelin-1 has profibrotic activities through the modulation of extracellular matrix deposition and turnover [88]. Data derived from a rat model of bleomycin-induced lung fibrosis suggested that bosentan could be beneficial for the treatment of IPF due to its ability to reduce collagen deposition in the lungs [89]. As such, a first randomized clinical trial was designed in order to assess the efficacy of bosentan in IPF [90]. The results of this trial did not show a benefit for the drug compared to placebo on the change of the exercise capacity. However, in a post hoc analysis a trend in favor of bosentan was shown with respect to the time to death or disease progression in a subgroup of patients diagnosed by surgical lung biopsy. These findings supported the design of a second large randomized clinical trial of bosentan in IPF. The trial has been recently completed and data are not yet available. However, the sponsor has released preliminary results of this trial; the primary end point of reduction in morbidity and mortality did not significantly differ between treatment and placebo.

A selective endothelin-A receptor antagonist (ambrisentan) was also under evaluation in the treatment of IPF patients, with or without associated pulmonary hypertension, in a randomized double-blind placebo-controlled trial. However, this trial has been terminated due to lack of efficacy according to an interim analysis of unblinded efficacy and safety data (see Press Releases on the company website, www.gilead.com). A further trial on this drug in patients with IPF and associated pulmonary hypertension has been terminated as well (www. clinicaltrials.gov). Full reports of these trials are awaited.

\subsection{Etanercept}

Etanercept is a soluble antagonist of TNF- $\alpha$; the molecule is recombinant human $\mathrm{p} 75$ TNF receptor and human IgG1 Fc portion fusion protein produced in a mammalian cell expression system and it was developed for the treatment of rheumatoid arthritis and other inflammatory conditions. 
The interest for the use of this drug in IPF was based on the observation of elevated levels of TNF- $\alpha$ in the lungs of animals in experimental models of lung fibrosis and in patients with IPF. TNF- $\alpha$ is a cytokine that displays profibrotic and inflammatory properties and the use of TNF- $\alpha$ antagonists was shown to inhibit pulmonary inflammation and fibrosis in an animal model of lung fibrosis. Given these observations, TNF- $\alpha$ was thought to be a potential target in the treatment of IPF and, therefore, a randomized placebo-controlled clinical trial in IPF patients was designed [91]. However, once again, promising preclinical data did not find confirmation in a randomized clinical trial: in fact, etanercept did not significantly affect the main efficacy end points. A nonsignificant reduction in disease progression was seen in several physiologic, functional and quality of life end points among subjects receiving etanercept [91].

\subsection{BIBF 1120}

Transmembrane receptor tyrosine kinases, a well-known pharmacological target in oncology, recently became the focus of clinical research in non-malignant disorders [92]. BIBF 1120 is a triple inhibitor of tyrosine kinase receptors, including PDGFR, VEGFR and fibroblast growth factor receptors. These receptors have been shown to be involved in lung fibrosis [93]. Treatment with BIBF 1120 was able to prevent the development of lung fibrosis in a bleomycin rat model [94]. Preliminary results from the Phase II TOMORROW study show that 12 months' treatment with BIBF1120 resulted in a clinically important reduction in the rate of decline in lung function in patients with IPF, compared to placebo. The $150 \mathrm{mg}$ twice daily dose of BIBF 1120 provided a $68 \%$ reduction in the rate of decline of FVC compared with placebo. Secondary end points supported the efficacy of BIBF1120. Only $2(2.3 \%)$ patients in the $150 \mathrm{mg}$ twice daily BIBF1120 group suffered from an acute exacerbation of the disease during the 12-month study period, compared with $12(13.8 \%)$ patients in the placebo group; in addition, patients taking $150 \mathrm{mg}$ twice daily BIBF1120 reported less deterioration in quality of life according to the St George's Respiratory Questionnaire compared with patients taking placebo. The total number of adverse events reported was similar in the BIBF1120 and placebo groups. This molecule is now entering Phase III in clinical development for the treatment of IPF.

\section{Current research goals and scientific rationale}

Because the absence of any reasonable effective drug for the treatment of IPF, a growing number of clinical trials have either been recently initiated or are in prospect (Table 1). However, these new studies are approaching some putative pathogenic mechanism by using a single drug approach. Because it is likely that several fibrogenic mechanisms/ pathways are playing a role in the development and progression of this disease, it is an easy prediction that no single agent will effectively reverse or even halt the fibrotic response in IPF. We need to search for multi-targeted approaches that should be more effective than single agents. Moreover, in the dynamic and complex process of fibrosis, it is currently unknown which upregulated or downregulated mediators are part of a pathogenic event and which might be epiphenomena. Therefore, despite remarkable and unprecedented progress in our understanding of cellular and molecular mechanisms of fibrosis progression, the translation of this knowledge into clinical practice through clinical trials has been disappointingly unsuccessful until now. One of the main problems is that a promising drug would have to show efficacy in at least two mechanistically distinct fibrosis models, with similar pathogenic mechanisms to IPF. Unfortunately, there is no experimental model of lung fibrosis that accurately mimics the disease, as we see it in human patients. In this context, different models of lung fibrosis have been developed over the years that mimic only few features of IPF. The most common of them is induced by intratracheal instillation or intravenous administration of bleomycin [95]. However, even though the bleomycin animal model offers valuable research tools for the elucidation of cells and mediators that can contribute to fibrotic changes it has significant limitations [95,96]. The main disadvantage of this experimental model is that it does not recapitulate the cardinal characteristics of IPF, primarily the progressive and irreversible nature of the human disease. In general, the fibrotic response in mice is self limiting and even reversible. Therefore, one of the most critical hallmarks of human IPF is not present in animals, which has to be considered when this model is used for drug intervention studies [95].

Finally, although the final goal of clinical research should ideally be to improve survival, this aim seems reasonably unrealistic to be obtained soon, mainly due to negative experience of large previous trials and the prohibitive costs related to large long-term trials dealing with rare diseases. However, there is still some lack of general consensus on which would be the best surrogate end point for mortality in IPF trials.

\section{Competitive environment}

For many years, the field of interstitial lung diseases was completely neglected by pharmaceutical companies worldwide. At the end of the 1990s, the initial report of an apparently extraordinary effect of a biological drug (IFN- $\gamma$ ) [81] on clinical course of IPF patients fuelled the interest of pharmaceutical companies for this neglected disease. Although these initial findings have been disproved after two large trials and huge financial investments, nonetheless, 20 years later, the landscape of clinical trials in IPF has totally changed. Now, not only many pharmaceutical private companies invest money and efforts to design and complete the 'perfect' trial, but also public health institutions (in particular the NIH in the US, through the IPFnet organization) promote large 


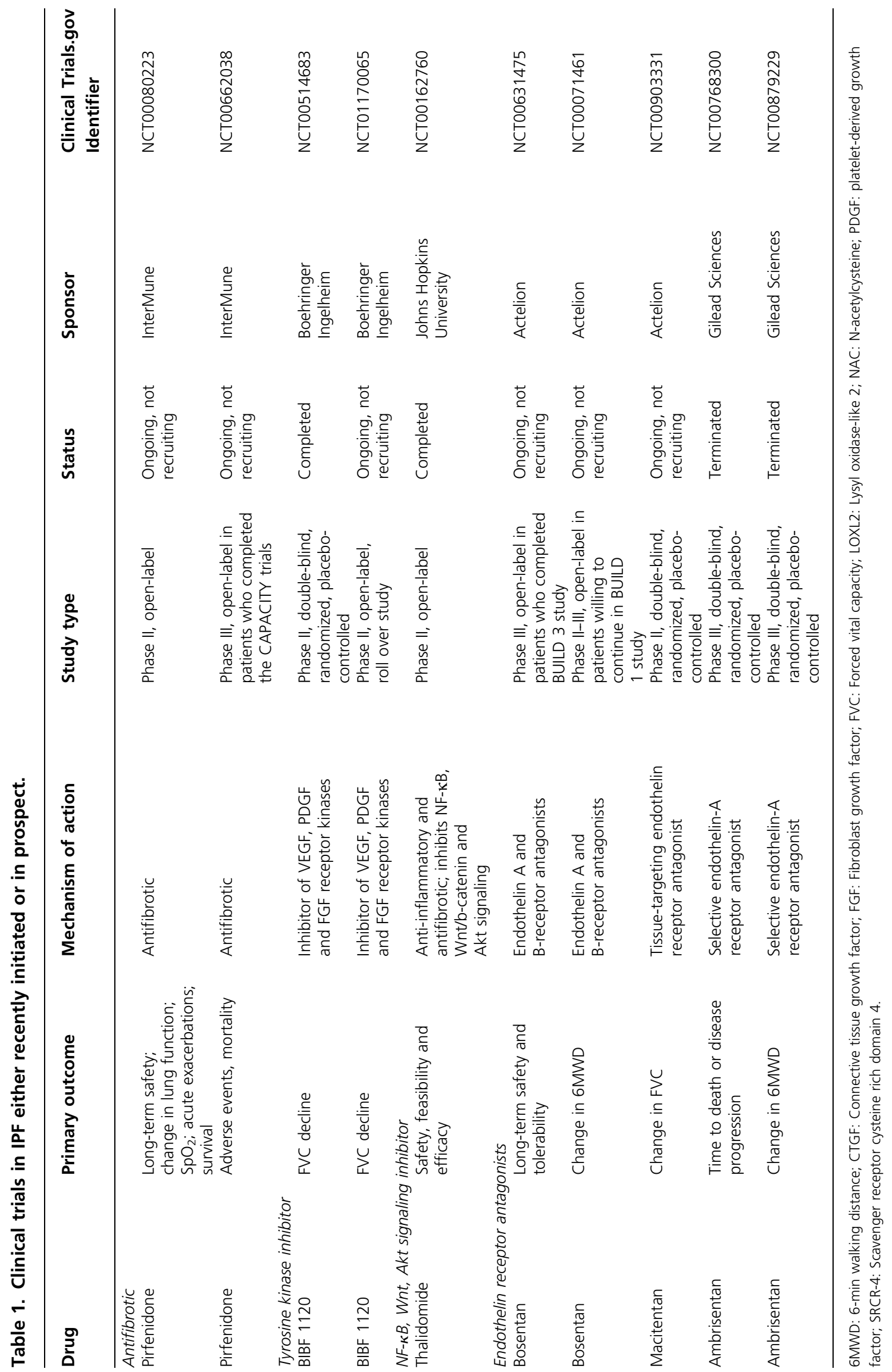




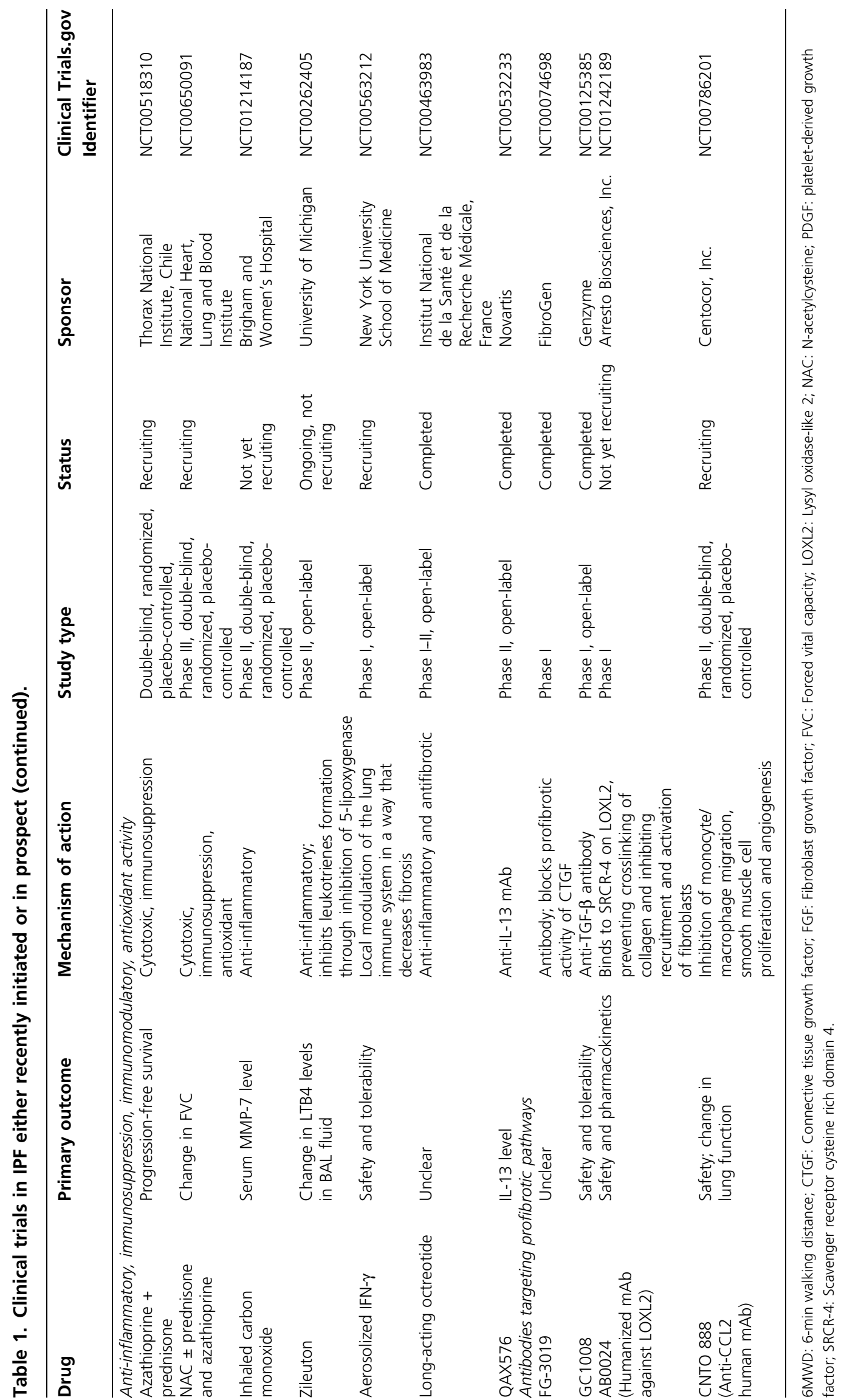




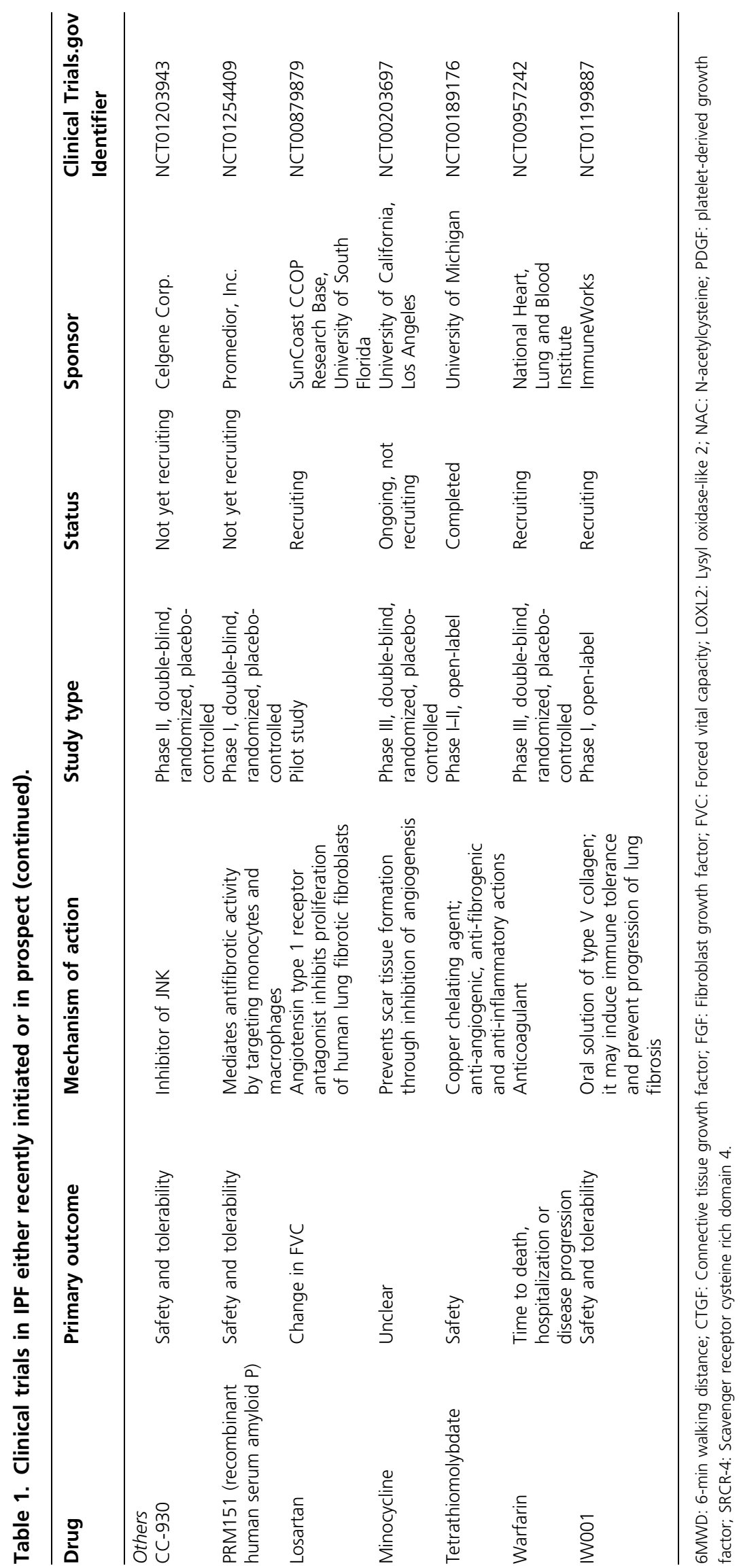


randomized trials to address important clinical questions arising from previous studies or to evaluate the efficacy of potentially relevant drugs, not supported by any company. Table 1 crystallizes the current landscape of clinical trials in IPF worldwide: all phases of clinical research are now represented and many, different geographical locations are present. On one hand, this is certainly good news for both IPF patients and for their physicians. On the other, however, competition for patient recruitment among different trials is now emerging: for this reason, companies started recently to approach areas of the world with a presumed high number of patients, but traditionally not part of clinical research networks (at least in this specific field), such as India and China: these 'big players', through excellent clinicians and top level health centers will play an increasingly important role in allowing completion of large trials in a timely schedule. Ideally, it would be advisable to have a global network of centers with expertise in IPF so that it will be feasible to complete large trials enrolling large numbers of patients with different racial background and followed in different professional environments. This will not only allow the completion of many trials (and, therefore, the testing of many different working hypotheses) but will also strength the collaboration within the IPF community at a global level.

\section{Potential development issues}

\subsection{Antioxidant drugs}

As mentioned above, the potential for beneficial effects on antioxidant therapies in IPF have been already explored by the IFIGENIA study evaluating the efficacy of NAC [67]. This study did not include a true placebo arm and, therefore, a further study on NAC is currently ongoing in the US to assess whether NAC alone can have any effect on the treatment of IPF patients.

However, new classes on antioxidant drugs are under evaluation for the treatment of IPF. In this context, NAPDH oxidase-4 (NOX-4, an enzyme that catalyze the reduction of $\mathrm{O}_{2}$ to reactive oxygen species) was found to be overexpressed in IPF lungs, particularly in the thickened wall of pulmonary arteries of IPF subjects [97,98]. In addition, NOX-4-dependent generation of reactive oxygen species seems to be required for TGF- $\beta 1$-induced myofibroblast differentiation and extracellular matrix production of IPF-derived mesenchymal cells $[97,99]$. As such, a novel class of orally available NOX-4 inhibitors might have the potential for being tested in clinical trials in IPF [100].

\subsection{Targeting the extracellular matrix}

Excessive extracellular matrix deposition is a key component of pathologic changes in IPF and results in an altered mechanic tension that supports the activation of pathogenic signaling pathways and tissue remodeling, including further activation of TGF- $\beta 1$, thus establishing a vicious circle that perpetuates the process of development of lung fibrosis [101].
As such, targeting the extracellular matrix could be a valuable approach in order to interfere with the mechanisms of lung fibrosis. Enzymes that modify the extracellular matrix include lysyl oxidases, a family of five enzymes sharing a conserved enzymatic domain with divergent $\mathrm{N}$ termini [102]. Lysyl oxidase (LOX) and LOXL2 promote crosslinking of fibrillar collagen, a major component of extracellular matrix in lung fibrosis. LOXL2 expression has been described in tumors and in liver fibrosis, with a possible role in promoting invasion. LOXL2 also appears to be overexpressed in lung tissue of patients with IPF, particularly in association with activated fibroblasts, reactive pneumocytes and vasculature in fibroblastic foci [103]. Moreover, treatment with a LOXL2 allosteric inhibitory $\mathrm{mAb}$ in the bleomycin-induced model of pulmonary fibrosis resulted in significant reduction of lung fibrosis, decreased number of activated fibroblasts in the lungs and significant reduction of profibrotic mediators, including TGF- $\beta 1$ [103].

Certainly, this mechanism provides a new appealing way of targeting lung fibrosis and, interestingly enough, the fact that this same pathway appears to be involved also in the process of tumor invasion by solid tumors could establish a further connection between fibrosis and cancer, thus, corroborating the recent thinking that effective treatment of IPF might take advantage from lessons learned in the oncology field [92].

\subsubsection{Is there a role for targeting the coagulation cascade?}

Accumulating evidence in recent years has provided a rationale for a beneficial role of targeting the coagulation cascade in the treatment of IPF. From a clinical point of view, there is only one unblinded study that addresses the effect of anticoagulant therapy in IPF patients. The study showed a significant reduction in overall mortality in the group of patients receiving prednisolone plus anticoagulation compared to the prednisolone alone group [104].

What are the mechanisms that would explain an implication of coagulation cascade in lung fibrosis? Several pieces of evidence have actually gained recent interest in supporting a potential role of the coagulation pathways in the fibroproliferative response to lung injury. First of all, the coagulation cascade appears to be activated in several fibrotic lung diseases by means of elevated expression of tissue factor in the lung $[105,106]$ and increased levels of thrombin in the bronchoalveolar lavage fluid of patients with fibrotic lung disease [106]. Moreover, Scotton et al. have provided evidence that the upstream coagulation zymogen, Factor X, is locally produced and activated in the intra-alveolar compartment of patients with IPF and in the bleomycin model [57]. In parallel, anticoagulant protein $\mathrm{C}$ pathway was shown to be deficient in IPF lungs. On the other hand, anticoagulants are highly effective in attenuating fibrosis in experimental animal models either prophylactically $[57,107]$ or therapeutically $[108]$.

Another mechanism that is related to the coagulation cascade and is thought to be involved in the process of fibrosis 
involves a direct receptor-mediated cellular effects elicited by activation of the major high-affinity thrombin receptor, proteinase-activated receptor (PAR)-1 [56]. Numerous cell types, including fibroblasts, epithelial cells and macrophages, express PAR-1. The activation of this receptor leads to the release of pro-inflammatory and profibrotic mediators such as PDGF and CTGF by fibroblasts [109,110]. In addition, it promotes the integrin-dependent TGF- $\beta 1$ activation [111] and thus contributes to the differentiation of fibroblasts into myofibroblasts. Furthermore, studies in the PAR-1 knockout mice have shown that these animals are protected from bleomycin-induced lung fibrosis, with an associated reduction in profibrotic mediators and chemokines [112]. Hopefully, this evidence will be soon translated into clinical application as several antagonists of PAR-1 are being already developed as drug compounds in the setting of cardiovascular disease. Few trials in this field have been already completed, although results are still awaited. A Phase II trial in patients undergoing non-urgent percutaneous coronary intervention has shown good tolerability and no increased risk of bleeding even when SCH 530348, an oral platelet protease-activated receptor-1 antagonist, was administered with aspirin or clopidogrel [113]. The potential advantages of PAR-1 antagonists compared to anticoagulant therapy are potentially elated to a reduced risk of bleeding complication as these novel drugs would compete with receptor-mediated cellular effects of coagulation proteinases rather than interfering with the coagulation cascade and fibrin formation.

\subsection{Is it possible to inhibit fibrocytes trafficking and differentiation, and the process of epithelial to mesenchymal transition?}

As mentioned, there are at least three potential sources for the increase of fibroblasts/myofibroblasts in the IPF lungs: the expansion of resident lung fibroblasts, the recruitment and differentiation of circulating mesenchymal precursors (fibrocytes) and the process of EMT.

In this context, a growing body of evidence suggests that some molecules can inhibit fibrocytes differentiation. For example, serum amyloid P (SAP), a naturally circulating soluble pattern recognition receptor, can directly inhibit monocytes from differentiating into fibrocytes [114]. Moreover, injections of SAP in rats or mice reduce bleomycininduced lung fibrosis [115]. Likewise, other putative regulators of fibrocytes include IFN- $\alpha_{2 b}$, adenosine A2A receptor and cysteinyl leukotriene receptor 1 [116-118]. Deletion or blockade of adenosine $\mathrm{A} 2 \mathrm{~A}$ receptor inhibited the recruitment of fibrocytes in bleomycin-treated mouse skin [117]. Likewise, fewer fibrocytes are noticed in the lungs of fluorescein isothiocyanate-treated 5-lipoxygenase-deficient mice compared with wild-type mice [118]. On the other hand, it is also possible to decrease the traffic of circulating fibrocytes to the lungs blocking the effect of the chemokines involved in their recruitment. Thus, it was demonstrated that treatment of bleomycin-exposed animals with specific neutralizing
anti-CXCL12 antibodies inhibited the intrapulmonary recruitment of circulating fibrocytes and attenuated lung fibrosis [119].

On the other hand, as previously mentioned, epithelial cells may respond to microenvironmental signals by undergoing EMT that also promotes the expansion of the myofibroblast population. In this context, targeting key signaling pathways to attenuate this process may have a profound effect on fibrogenesis and, currently, several studies are evolving in the area of cancer [120]. Thus, a recent report has shown that sorafenib (Nexavar; Bayer HealthCare Pharmaceuticals, Inc.), a VEGFR and PDGFR- $\beta$ inhibitor with activity against RAF kinase, exerts a potent inhibitory activity against the EMT by inhibiting MAPK signaling and SNAI1 expression in hepatocellular carcinoma cells [121]. Some microRNAs (miRNAs) could be other targets by which to block EMT (see below).

\subsection{Is it possible to improve epithelial regeneration?}

Three members of the fibroblast growth factor (FGF) family, keratinocyte growth factor (KGF), FGF-10 and FGF-1, as well as the hepatocyte growth factor (HGF), may play a role for the appropriate epithelial repair in IPF lungs. For example, it has been shown that transient overexpression of gremlin, a BMP antagonist, induces alveolar epithelial apoptosis and lung fibrosis that is reverted after the selective upregulation of FGF10 [122]. Likewise, it has been demonstrated that FGF-1 displays antifibrotic functions downregulating collagen expression and antagonizing some profibrotic effects of TGF- $\beta$ [123,124]. More recently, it was reported that FGF-1 reverts TGF- $\beta 1$-induced EMT in alveolar epithelial cells throughout MEK-ERK pathway inducing the dephosphorylation of Smad2 [125].

Also, experimental studies have shown that human HGF gene transfer increases endogenous lung HGF preventing AECIIs from further apoptosis and resulting in reduced fibrosis after bleomycin injury [126]. Likewise, intratracheal instillation or intravenous administration of KGF protects the lungs against several experimental injuries [127]. More recently, it was shown that bone marrow stem cells expressing KGF via an inducible lentivirus attenuates bleomycin-induced pulmonary fibrosis [128]. In addition, KGF-gene transduction by intratracheal administration of KGF-expressing adenovirus vector was shown to result in decreased fibrosis in the bleomycin model in mice and reduced mortality. KGF-associated effects included alveolar epithelial cell proliferation, suppression of TGF- $\beta 1$ production and increased SP secretion [129].

Whether these approaches will have a role in the treatment of IPF is at the present largely unclear. However, it has to be mentioned that at least one molecule of the FGF family has reached the clinical use for epithelial repair in other fields. In fact, human recombinant KGF (palifermin, Kepivance ${ }^{\circledR}$ ) is marketed in some countries for the prevention of severe oral mucositis in patients with hematologic malignancies 
receiving myelotoxic therapy requiring hematopoietic stem cell support [130-132].

\subsection{Interfering with developmental pathways}

In a recent report, using the bleomycin-induced model of pulmonary fibrosis in transgenic BAT-gal mice, it was demonstrated that, as reported in IPF, aberrant activation of Wnt signaling in the lungs was induced after injury [133]. Bleomycin-induced fibrosis and the overexpression of profibrotic genes were significantly reduced by the specific inhibitor of Wnt/ $\beta$-catenin/CBP-driven transcription, ICG-001. The inhibitor decreased epithelial cell apoptosis and EMT both in vivo and in vitro. Amelioration of fibrosis, together with improved survival, following both concurrent and late administration of ICG-001, provided preliminary evidence for selective blockade of Wnt/ $\beta$-catenin-CBP-dependent transcription signaling as a potential therapeutic strategy for human fibrotic lung diseases.

\subsection{Embryonic stem cells}

Because the current strategies for IPF treatment have been limited and unsuccessful, novel therapeutic strategies are urgently needed. Lung transplantation is usually the only option for patients with irreversible structural lung damage including those with IPF; however, organ availability lags far behind demand. Thus, given the scarcity of donors and the aging and disease complications of IPF patients, transplantation is indicated only in carefully selected patients. In addition, many of them die in the waiting list. The main problem after injury is to re-establish the integrity and functional organization of the epithelial layer, and of the alveolar-capillary units. Probably this occurs during normal repair by the migration and spreading of nearby and newly recruited circulating progenitor cells that proliferate and undergo phenotypic differentiation to cover the denuded surfaces [134]. In this context, a number of studies performed in the last decade have suggested that both embryonic and adult tissue-derived stem cells may play a role in the regeneration and repair of diseased adult organs including the lungs [135].

Embryonic stem cells (ESCs) are self-renewing pluripotent cells which can be induced to differentiate into a wide range of different cell types [136]. Thus, at least theoretically, alveolar type II epithelial cells derived from ESCs may be used therapeutically to treat fibrotic disorders that show lost of epithelial integrity and abnormalities in re-epithelialization. However, an important goal in this area is to obtain pure AECIIs from embryonic cells. This objective was recently achieved [137], and it was also demonstrated that lung progenitor epithelial cells derived from human ESCs can indeed be transplanted into damaged alveoli of mice and that these cells are able to reverse bleomycin-induced pathological changes [138]. Thus, while non-treated mice exposed to bleomycin showed large extent of interstitial thickening by extensive interstitial inflammation and fibrosis, with the formation of cystic air spaces, ESC-AECIIs transplanted mice exhibited a greatly reduced damage as evidenced by only a few isolated, small areas of injured tissue surrounded by much larger areas of normal alveolar structure [138]. Whether this effect resulted from structural engraftment of the administered cells or reflected some kind of paracrine effect of the ESC-derived AECIIs is not yet clear. Interestingly, the therapeutic benefits provided by the transplantation of these cells were long-term and without the development of teratomas [138]. This is an important observation because one of the potential risks with the use of ESCs or induced pluripotent stem cells is the induction of malignancies.

\subsection{Induced-pluripotent stem cells}

Recently, a novel dedifferentiation technology that allows the generation of induced- pluripotent stem (iPS) cells has been developed. The expression of four transcription factors Oct4, Sox2, c-myc and Klf4 is able to induce epigenetic reprogramming of a somatic genome to an embryonic pluripotent state $[139,140]$. Patient-adapted iPS cells remove the major problem of donor immune rejection for clinical applications and also mitigate ethical concerns associated with the use of human ESCs [141]. However, the use of retrovirus-transduced oncogenes represents a serious barrier to the eventual use of reprogrammed cells for therapeutic application.

In addition, although several methods have been developed for generating iPS cells free of reprogramming transgenes, a sufficiently efficient reprogramming system is still required in order to achieve the widespread derivation of diseasespecific iPS from humans with different disorders. In this context, Somers et al. [142] reported the use of a humanized version of a single lentiviral 'stem cell cassette' vector in order to accomplish efficient reprogramming of normal or diseased skin fibroblasts obtained from humans of virtually any age. The vast majority of clones obtained with this method contained a single integrated vector copy which can be excised to obtain human iPS cells free of integrated transgenes. The authors applied this strategy to generate numerous lung disease-specific iPS cell lines from individuals with a variety of diseases affecting the epithelial, endothelial or interstitial compartments of the lung, including cystic fibrosis, $\alpha-1$ antitrypsin deficiency-related emphysema and scleroderma. This work indicates that it is possible to reprogram human somatic cells with high efficiency by using a single polycistronic excisable lentivirus expressing the four transcription factors, Oct4, Klf4, Sox2 and c-myc. Additionally, the authors demonstrated that human iPSC generated with this approach have the ability to robustly differentiate into definitive endoderm in vitro, the developmental precursor tissue of lung epithelia. However, several problems should be solved, for example, their capacity for tumorigenesis in vivo, in order to determine whether iPS cells will have a role in the treatment of chronicdegenerative diseases, including IPF, as vehicles for gene or cell therapies. 


\subsection{Adult mesenchymal stem cells}

Mesenchymal stem cells (MSCs) are multipotent cells able to differentiate into a range of cell types that are being tested for their regenerative potential in several diseases. In a pioneer work, Ortiz et al. [143] demonstrated that MSCs engraft in the lungs of normal mice at low levels, but engraftment increases significantly in response to bleomycin-induced lung damage. In the injured lungs, engrafted donor cells adopted an epithelium-like morphology. In addition, it was found that early but not late administration of MSCs ameliorated the fibrotic response observed in the lungs of bleomycintreated mice. In another study, it was indicated that bone marrow-derived MSCs seem to decrease bleomycin-induced lung injury and fibrosis not only by supplying stem cells to the lung but also because they produce growth factors, such as GM-CSF and G-CSF that may mobilize endogenous stem cells from bone marrow pools to the lungs [144]. Interestingly, in this study, donor-derived cells in the lungs showed phenotypic characteristics of several lung cell types, including type I and II alveolar epithelial cells, fibroblasts and endothelial cells.

More recently, MSCs derived from the Wharton's jelly of human umbilical cords were used to prevent bleomycininduced fibrosis [145]. These cells have the advantage of ready availability and that do not require invasive bone marrow biopsies. As previously shown, the pneumonitis induced by bleomycin was strongly attenuated by MSC treatment. Interestingly, MSCs were detected in both the fibrotic and nonfibrotic areas of the bleomycin-injured lungs at 14 days but not at 28 days after injection. The antifibrotic effect appears to be related to downregulation of lung cytokines and TIMP expression. Likewise, in another recent study it was shown that systemic transfer of MSCs effectively reduced the bleomycin-induced lung injury and fibrosis through the downregulation of NO metabolites, and pro-inflammatory and angiogenic cytokines [146].

In general, the results obtained with adult MSCs are promising. However, a number of yet unresolved problems persist. Several studies using different technical approaches have shown virtually no detectable reconstitution of lung alveolar epithelial cells by bone marrow derived cells [147,148]. Nevertheless, chronic or progressive lung injury may result in more substantial engraftment of stem cells. After systemic adult stem cells administration, many of them may initially localize in lungs as the first major capillary bed encountered, and lung damage may result in increased retention of these cells. The phenotypic instability related to plasticity raises concerns the outcome of transplanted cells that may undergo unwanted and deleterious differentiation after implantation/ engraftment at specific sites [149]. The transformation potential is also a major concern $[150,151]$. In addition, it is important to consider that the mechanisms by which stem cells acquire one or another phenotype(s) in the lung remain poorly understood. Moreover, mechanisms responsible for its therapeutic role are not well understood, and may involve plasticity or, as much evidence indicates, paracrine and immunomodulatory activities without significant engraftment and differentiation [151].

\subsection{Bioengineering the lung}

In the last years, several studies have tried to bioengineer lung-like tissues using synthetic (such as polymers, polyglycolic acid) or natural (e.g., collagen, basement membrane components) scaffolds on which to grow lung (primarily epithelial) cells, but these scarcely replicate the complexity of the lungs. In a recent paper, it was shown that mouse lungs can successfully be decellularized while maintaining the extracellular matrix in the correct geospatial branching pattern even after prolonged ventilation [152]. Moreover, fetal lung cells were successfully infused and cultured in decellularized, ventilated lungs. This work demonstrated that decellularized whole lungs can be used as natural scaffolds in a bioreactor system, supporting the fact that the natural matrix of the lung is an excellent scaffold from which to rebuild a lung.

In the same line of thought, an outstanding work dealing with the construction of a functional tissue-engineered lung, using rat as a model system, was also recently published [153]. The authors decellularized native lung tissue in order to remove all immunogenic cellular constituents, showing that after this procedure, the tissue retained its alveolar micro-architecture, its ability to function as a barrier to particulates and its tissue mechanics. The acellular lung matrix was repopulated with mixed populations of neonatal lung epithelial cells, which resulted in regional-specific epithelial seeding in correct anatomic locations. Likewise, microvascular lung endothelial cells injected into the pulmonary artery of acellular scaffolds were able to adhere throughout the scaffold vasculature. To enhance the survival and differentiation of lung epithelium, the matrix was cultured in a bioreactor designed to mimic certain features of the fetal lung environment, including vascular perfusion and liquid ventilation. Finally, to determine whether engineered rodent lungs were implantable and functional for gas exchange, orthotopic left lung transplantation was performed in four rats. All engineered lungs became perfused with blood over a period of seconds to minutes, and blood gas analysis revealed that the tissue-engineered lungs were effective in exchanging oxygen and carbon dioxide. Although yet far from human tissue bioengineering, these results are encouraging.

\subsection{0 microRNAs}

miRNAs are small (17 - 25 nucleotides) non-coding, naturally occurring RNAs that comprise a new class of transcriptional gene regulators. miRNA guide post-transcriptional gene silencing by either translational repression or by degradation.

Dysregulation of miRNAs is known to be involved in a variety of pathological processes, and three recent papers have suggested a possible role in the pathogenesis of IPF. 
Pandit et al. [154] performed miRNA microarrays in $10 \mathrm{IPF}$ and 10 control lungs and found 46 miRNAs differentially expressed. Among the significantly decreased miRNAs in IPF lungs were let-7d, miR-26 and several members of the miR-30 family. Decreased let-7d was primarily localized in epithelial cells and directly inhibited by TGF- $\beta$. Importantly, the inhibition of let-7d in vitro caused EMT-like changes in lung epithelial cells, while in vivo provoked spontaneous alveolar septal thickening in the mouse lungs.

Likewise, it was reported that lungs of mice with bleomycin-induced fibrosis as well as IPF lungs show an upregulation of miR-21 [155]. Increased miR-21 expression was primarily localized to myofibroblasts. Increasing miR-21 levels promoted, whereas knocking down miR-21 attenuated, the pro-fibrogenic activity of TGF- $\beta 1$ in fibroblasts, and miR-21 antisense probes attenuated bleomycin-induced lung fibrosis.

More recently, in a large scale screening for miRNAs potentially involved in bleomycin-induced fibrosis, it was found that the expression of miR-29, which is expressed by mesenchymal cells, is significantly reduced in fibrotic lungs [156]. miR-29 levels inversely correlated with the expression of several profibrotic target genes and with the severity of the fibrosis. In fibroblasts, miR-29 was suppressed by TGF $\beta 1$ and many fibrosis-associated genes upregulated by TGF $\beta 1$ were derepressed by miR-29 knockdown.

Taken together, these studies suggest that, at least in part, the effects of TGF- $\beta$, an essential pathological mediator of lung fibrosis, may be related to its capacity to decrease the expression of 'antifibrogenic' miRNAs in alveolar epithelial cells (let-7d) or fibroblasts (miR-29), while may increase the expression of a 'profibrogenic' miRNA (miR-21) in lung fibroblasts.

The putative therapeutic application of miRNAs involves two strategies [157]. One approach is directed to inhibit upregulated, potentially pathogenic, miRNAs by using miRNA antagonists, such as anti-miRs, locked-nucleic acids or antagomiRs. These miRNA antagonists are oligonucleotides with sequences complementary to the endogenous miRNA. The second strategy, miRNA replacement, involves the reintroduction of a downregulated miRNA attempting to restore a loss of function [157].

Some advances have been made in cancer treatment. For example, let-7 expression is reduced in a subset of NSCLC patients, and this reduction is correlated with poor prognosis. Using both inducible and constitutive expression systems, substantial tumor suppression by let- $7 \mathrm{~g}$ that function as a tumor suppressor both in xenografts and in a mouse lung tumor model has been found [158]. These findings make clinically relevant predictions related to the use of let-7-based therapeutic agents in this type of cancer.

Another miRNA that is also suppressed in human NSCLC tissues is miR-34a, and recent data provide evidence for the safe and effective therapeutic delivery of a synthetic miR-34a mimic [159]. It was demonstrated that miR-34a inhibits the growth of cultured lung cancer cells and that intratumoral delivery of formulated miR-34a in a lipid-based vehicle blocks lung tumor growth in mice.

In lung fibrosis, it was shown that administration of miR-21 antisense probes attenuated the severity of bleomycininduced lung fibrosis in mice, even when treatment was started $5-7$ days after initiation of the lung injury, suggesting the possibility of using miRNA therapeutics [155].

Certainly, many studies are needed to address long-term efficacy and safety of miRNA therapy in humans but the current evidence supports the development of this new class of therapeutics.

\section{Expert opinion}

The treatment of IPF represents one of the greatest challenges confronting respiratory medicine and, currently, there is no effective therapeutic option for IPF. Perhaps some of the drugs that are under evaluation in clinical trials will slow the decline of the pulmonary function tests or hopefully stabilize some patients. Nonetheless, it appears clear that new therapeutic approaches are urgently needed.

A growing list of mediators and pathways putatively involved in the pathogenesis of IPF could be exploited in the development of antifibrotic drugs. However, although extensive progress has been made in identifying the pathogenic mechanisms implicated in this disease, many unanswered questions remain. In this context, the lack of efficient therapeutic options and design of more effective drugs is at least partially due to the fact that the underlying mechanisms leading to lung fibrosis are not completely understood.

In addition, the design of antifibrotic strategies for clinical purposes entails enormous obstacles. One of the most difficult problems that we face is to find the most appropriate clinical end points: ideally, the best are survival and quality of life. An effective drug should, therefore, significantly decrease mortality, improving at the same time the quality of life. However, this scenario implies long-term large randomized clinical trials with several hundred or even thousands of well-characterized patients. Unfortunately, noninvasive methods, such as serum biomarkers or better imaging or respiratory functional techniques that can quickly quantify changes in the natural history of the disease, for example, rapid versus slowly progressive disease are scanty. This is an important issue because IPF is a heterogeneous disease showing a varied clinical behavior with some patients evolving slowly while others progress rapidly and die in few months or years after the beginning of symptoms.

Given the complexity of this disease, it would be expected that the best therapeutic approach should include several drugs that target epithelial cells and fibroblasts and several signaling pathways. Disappointingly, however, a recent trial with imatinib that potentially targets several signaling pathways by inhibiting transmembrane receptor tyrosine kinases showed no effect. 
Is there a promissory future for the treatment of IPF? Yes, but not in the short time. Regenerative therapy using stem cells, lung bioengineering to obtain healthy organs for transplant or the utilization of nucleic acid-based therapeutic molecules (e.g., miRNA) can open solid perspectives and it is hoped that combining these and other modalities will translate into improved clinical outcomes. Certainly, the accurate scientific appraisal of potential risks and limitations of these therapies to generate strategies for overcoming them and providing a safe and efficient clinical application will be necessary.

\section{Declaration of interest}

The authors state no conflict of interest and have received no payment in preparation of this manuscript. M Selman is member of the Steering Committee and Consultant of Boehringer Ingelheim for the BIBF-1120 protocol. L Richeldi is member of the Steering Committee and Consultant of Boehringer Ingelheim for BIBF-1120 protocol and provided consultation for Gilead and Celgene regarding IPF-related studies.

\section{Bibliography}

Papers of special note have been highlighted as either of interest $(\bullet)$ or of considerable interest

$(\bullet \bullet)$ to readers.

1. Raghu G, Weycker D, Edelsberg J, et al. Incidence and prevalence of idiopathic pulmonary fibrosis. Am J Respir Crit Care Med 2006;174:810-16

2. Gribbin J, Hubbard RB, Le Jeune I, et al. Incidence and mortality of idiopathic pulmonary fibrosis and sarcoidosis in the UK. Thorax 2006;61:980-5

3. Noth I, Martinez FJ. Recent advances in idiopathic pulmonary fibrosis. Chest 2007;132:637-50

4. Selman M, Rojas M, Mora AL, Pardo A. Aging and interstitial lung diseases: unraveling an old forgotten player in the pathogenesis of lung fibrosis.

Semin Respir Crit Care Med 2010;31:607-17

5. Olson AL, Swigris JJ, Lezotte DC, et al. Mortality from pulmonary fibrosis increased in the United States from 1992 to 2003. Am J Respir Crit Care Med 2007;176:277-84

6. Selman M, Carrillo G, Estrada A, et al. Accelerated variant of idiopathic pulmonary fibrosis: clinical behavior and gene expression pattern. PLoS One 2007;2:e482

7. Collard HR, Moore BB, Flaherty KR, et al. Acute exacerbations of idiopathic pulmonary fibrosis. Am J Respir Crit Care Med 2007;176:636-43

8. Song JW, Hong SB, Lim CM, et al. Acute exacerbation of idiopathic pulmonary fibrosis: incidence, risk factors, and outcome. Eur Respir J 2010; 37(2):356-63

9. Boon K, Bailey NW, Yang J, et al. Molecular phenotypes distinguish patients with relatively stable from progressive idiopathic pulmonary fibrosis (IPF). PLoS One 2009; $4:$ e5134

10. Cottin V, Nunes H, Brillet PY, et al. Combined pulmonary fibrosis and emphysema: a distinct underrecognised entity. Eur Respir J 2005;26:586-93

11. Mejia M, Carrillo G, Rojas-Serrano J, et al. Idiopathic pulmonary fibrosis and emphysema: decreased survival associated with severe pulmonary arterial hypertension. Chest 2009;136:10-15

12. Cottin V, Le Pavec J, Prevot G, et al. Pulmonary hypertension in patients with combined pulmonary fibrosis and emphysema syndrome. Eur Respir J 2010;35:105-11

13. Harris JM, Johnston ID, Rudd R, et al. Cryptogenic fibrosing alveolitis and lung cancer: the BTS study. Thorax 2010;65:70-6

14. Gotway MB, Freemer MM, King TE Jr. Challenges in pulmonary fibrosis. 1: Use of high resolution CT scanning of the lung for the evaluation of patients with idiopathic interstitial pneumonias. Thorax 2007;62:546-53

15. Katzenstein AL, Mukhopadhyay S, Myers JL. Diagnosis of usual interstitial pneumonia and distinction from other fibrosing interstitial lung diseases. Hum Pathol 2008;39:1275-94

16. Lawson WE, Loyd JE. Will the genes responsible for familial pulmonary fibrosis provide clues to the pathogenesis of IPF? Am J Respir Crit Care Med 2010;182:1342-3

17. Raghu G, Collard HR, Egan JJ, et al. Idiopathic pulmonary fibrosis: evidence based guidelines for diagnosis and management. Am J Respir Crit Care Med 2011; In press

18. Taskar VS, Coultas DB. Is idiopathic pulmonary fibrosis an environmental disease? Proc Am Thorac Soc 2006;3:293-8

19. Steele MP, Speer MC, Loyd JE, et al. Clinical and pathologic features of familial interstitial pneumonia. Am J Respir Crit Care Med 2005;172:1146-52

20. Hubbard R, Lewis S, Richards K, et al. Occupational exposure to metal or wood dust and aetiology of cryptogenic fibrosing alveolitis. Lancet 1996;347:284-9

21. Baumgartner KB, Samet JM, Coultas DB, et al. Occupational and environmental risk factors for idiopathic pulmonary fibrosis: a multicenter case-control study. Collaborating Centers. Am J Epidemiol 2000;152:307-15

22. Selman M, King TE, Pardo A. Idiopathic pulmonary fibrosis: prevailing and evolving hypotheses about its pathogenesis and implications for therapy. Ann Intern Med 2001;134:136-51

23. Selman M, Pardo A. Role of epithelial cells in idiopathic pulmonary fibrosis: from innocent targets to serial killers. Proc Am Thorac Soc 2006;3:364-72

24. Strieter RM. What differentiates normal lung repair and fibrosis? Inflammation, resolution of repair, and fibrosis. Proc Am Thorac Soc 2008;5:305-10

25. Strieter RM, Mehrad B. New mechanisms of pulmonary fibrosis. Chest 2009;136:1364-70

26. Gilani SR, Vuga LJ, Lindell KO, et al. CD28 down-regulation on circulating CD4 T-cells is associated with poor prognoses of patients with idiopathic pulmonary fibrosis. PLoS One 2010;5:e8959

27. Zuo F, Kaminski N, Eugui E, et al. Gene expression analysis reveals matrilysin as a key regulator of pulmonary fibrosis in mice and 
humans. Proc Natl Acad Sci USA 2002;99:6292-7

28. Wilson MS, Madala SK, Ramalingam TR, et al. Bleomycin and IL-1beta-mediated pulmonary fibrosis is IL-17A dependent. J Exp Med 2010;207:535-52

29. Lo Re S, Dumoutier L, Couillin I, et al. IL-17A-producing gammadelta $\mathrm{T}$ and Th17 lymphocytes mediate lung inflammation but not fibrosis in experimental silicosis. J Immunol 2010;184:6367-77

30. Myers JL, Katzenstein AL. Epithelial necrosis and alveolar collapse in the pathogenesis of usual interstitial pneumonia. Chest 1988;94:1309-11

31. Uhal BD, Joshi I, Hughes WF, et al. Alveolar epithelial cell death adjacent to underlying myofibroblasts in advanced fibrotic human lung. Am J Physiol 1998;275:L1192-9

32. Barbas-Filho JV, Ferreira MA, Sesso A, et al. Evidence of type II pneumocyte apoptosis in the pathogenesis of idiopathic pulmonary fibrosis (IFP)/usual interstitial pneumonia (UIP). J Clin Pathol 2001;54:132-8

33. Thannickal VJ, Horowitz JC. Evolving concepts of apoptosis in idiopathic pulmonary fibrosis. Proc Am Thorac Soc 2006;3:350-6

34. Nogee LM, Dunbar AE III, Wert SE, et al. A mutation in the surfactant protein $\mathrm{C}$ gene associated with familial interstitial lung disease. N Engl J Med 2001;344:573-9

35. van Moorsel $\mathrm{CH}$, van Oosterhout $\mathrm{MF}$, Barlo NP, et al. Surfactant protein C mutations are the basis of a significant portion of adult familial pulmonary fibrosis in a dutch cohort. Am J Respir Crit Care Med 2010;182:1419-25

36. Wang WJ, Mulugeta S, Russo SJ, Beers MF. Deletion of exon 4 from human surfactant protein $\mathrm{C}$ results in aggresome formation and generation of a dominant negative. J Cell Sci 2003;116:683-92

37. Mulugeta $S$, Nguyen V, Russo SJ, et al. A surfactant protein $\mathrm{C}$ precursor protein BRICHOS domain mutation causes endoplasmic reticulum stress, proteasome dysfunction, and caspase 3 activation. Am J Respir Cell Mol Biol 2005;32:521-30
38. Korfei M, Ruppert C, Mahavadi P, et al. Epithelial endoplasmic reticulum stress and apoptosis in sporadic idiopathic pulmonary fibrosis. Am J Respir Crit Care Med 2008;178:838-46

39. Lawson WE, Crossno PF, Polosukhin VV, et al. Endoplasmic reticulum stress in alveolar epithelial cells is prominent in IPF: association with altered surfactant protein processing and herpesvirus infection. Am J Physiol Lung Cell Mol Physiol 2008;294:L1119-26

40. Vannella KM, Moore BB. Viruses as co-factors for the initiation or exacerbation of lung fibrosis. Fibrogenesis Tissue Repair 2008;1:2

41. Armanios MY, Chen JJ, Cogan JD, et al. Telomerase mutations in families with idiopathic pulmonary fibrosis. N Engl J Med 2007;356:1317-26

42. Tsakiri KD, Cronkhite JT, Kuan PJ, et al. Adult-onset pulmonary fibrosis caused by mutations in telomerase. Proc Natl Acad Sci USA 2007;104:7552-7

43. Alder JK, Chen JJ, Lancaster L, et al. Short telomeres are a risk factor for idiopathic pulmonary fibrosis. Proc Natl Acad Sci USA 2008;105:13051-6

44. Waisberg DR, Barbas-Filho JV, Parra ER, et al. Abnormal expression of telomerase/apoptosis limits type II alveolar epithelial cell replication in the early remodeling of usual interstitial pneumonia/idiopathic pulmonary fibrosis. Hum Pathol 2010;41:385-91

45. Andersson-Sjoland A, de Alba CG, Nihlberg K, et al. Fibrocytes are a potential source of lung fibroblasts in idiopathic pulmonary fibrosis. Int J Biochem Cell Biol 2008;40:2129-40

46. Herzog EL, Bucala R. Fibrocytes in health and disease. Exp Hematol 2010;38:548-56

47. Keeley EC, Mehrad B, Strieter RM. Fibrocytes: bringing new insights into mechanisms of inflammation and fibrosis. Int J Biochem Cell Biol 2010;42:535-42

48. Garcia-de-Alba C, Becerril C, Ruiz V, et al. Expression of matrix metalloproteases by fibrocytes: possible role in migration and homing. Am J Respir Crit Care Med 2010;182:1144-52
49. Hinz B, Phan SH, Thannickal VJ, et al. The myofibroblast: one function, multiple origins. Am J Pathol 2007;170:1807-16

50. Willis BC, Liebler JM, Luby-Phelps K, et al. Induction of epithelial-mesenchymal transition in alveolar epithelial cells by transforming growth factor-beta1: potential role in idiopathic pulmonary fibrosis. Am J Pathol 2005;166:1321-32

51. Kim KK, Kugler MC, Wolters PJ, et al. Alveolar epithelial cell mesenchymal transition develops in vivo during pulmonary fibrosis and is regulated by the extracellular matrix. Proc Natl Acad Sci USA 2006;103:13180-5

52. Willis BC, Borok Z. TGF-beta-induced EMT: mechanisms and implications for fibrotic lung disease. Am J Physiol Lung Cell Mol Physiol 2007;293:L525-34

53. Sides MD, Klingsberg RC, Shan B, et al. The Epstein-Barr Virus LMP 1 and TGF- $\{$ beta 1 Synergistically Induce EMT in Lung Epithelial Cells. Am J Respir Cell Mol Biol 2010

54. Humphreys BD, Lin SL, Kobayashi A, et al. Fate tracing reveals the pericyte and not epithelial origin of myofibroblasts in kidney fibrosis. Am J Pathol 2010;176:85-97

55. Pardo A, Selman M. Matrix metalloproteases in aberrant fibrotic tissue remodeling. Proc Am Thorac Soc 2006;3:383-8

56. Chambers RC. Procoagulant signalling mechanisms in lung inflammation and fibrosis: novel opportunities for pharmacological intervention? $\mathrm{Br} \mathrm{J}$ Pharmacol 2008;153 Suppl 1:S367-78

57. Scotton CJ, Krupiczojc MA, Konigshoff M, et al. Increased local expression of coagulation Factor X contributes to the fibrotic response in human and murine lung injury. J Clin Invest 2009;119:2550-63

58. Selman M, Pardo A, Kaminski N. Idiopathic pulmonary fibrosis: aberrant recapitulation of developmental programs? PLoS Med 2008;5:e62

59. Konigshoff M, Balsara N, Pfaff EM, et al. Functional Wnt signaling is increased in idiopathic pulmonary fibrosis. PLoS One 2008;3:e2142 
60. Konigshoff M, Kramer M, Balsara N, et al. WNT1-inducible signaling protein-1 mediates pulmonary fibrosis in mice and is upregulated in humans with idiopathic pulmonary fibrosis.

J Clin Invest 2009;119:772-87

61. Koli K, Myllarniemi M, Vuorinen K, et al. Bone morphogenetic protein- 4 inhibitor gremlin is overexpressed in idiopathic pulmonary fibrosis. Am J Pathol 2006; 169:61-71

62. American Thoracic Society. Idiopathic pulmonary fibrosis: diagnosis and treatment. International consensus statement. American Thoracic Society (ATS), and the European Respiratory Society (ERS). Am J Respir Crit Care Med 2000;161:646-64

63. Richeldi L, Davies HR, Ferrara G, Franco F. Corticosteroids for idiopathic pulmonary fibrosis. Cochrane Database Syst Rev 2003:CD002880. Edited; published in Issue 2, 2010

64. Spagnolo P, Del Giovane C, Luppi F, et al. Non-steroid agents for idiopathic pulmonary fibrosis. Cochrane Database Syst Rev 2010:CD003134

65. Raghu G, Depaso WJ, Cain K, et al. Azathioprine combined with prednisone in the treatment of idiopathic pulmonary fibrosis: a prospective double-blind, randomized, placebo-controlled clinical trial. Am Rev Respir Dis 1991;144:291-6

66. Tirouvanziam R, Conrad CK, Bottiglieri T, et al. High-dose oral $\mathrm{N}$-acetylcysteine, a glutathione prodrug, modulates inflammation in cystic fibrosis. Proc Natl Acad Sci USA 2006; 103:4628-33

67. Demedts M, Behr J, Buhl R, et al. High-dose acetylcysteine in idiopathic pulmonary fibrosis. N Engl J Med 2005;353:2229-42

68. Behr J, Demedts M, Buhl R, et al. Lung function in idiopathic pulmonary fibrosis-extended analyses of the IFIGENIA trial. Respir Res 2009;10:101

69. Iyer SN, Gurujeyalakshmi G, Giri SN. Effects of pirfenidone on transforming growth factor-beta gene expression at the transcriptional level in bleomycin hamster model of lung fibrosis. J Pharmacol Exp Ther 1999;291:367-73
70. Iyer SN, Gurujeyalakshmi G, Giri SN. Effects of pirfenidone on procollagen gene expression at the transcriptional level in bleomycin hamster model of lung fibrosis. J Pharmacol Exp Ther 1999;289:211-18

71. Nakazato H, Oku H, Yamane S, et al. A novel anti-fibrotic agent pirfenidone suppresses tumor necrosis factor-alpha at the translational level. Eur J Pharmacol 2002;446:177-85

72. Oku H, Nakazato H, Horikawa T, et al. Pirfenidone suppresses tumor necrosis factor-alpha, enhances interleukin-10 and protects mice from endotoxic shock. Eur J Pharmacol 2002;446:167-76

73. Raghu G, Johnson WC, Lockhart D, Mageto Y. Treatment of idiopathic pulmonary fibrosis with a new antifibrotic agent, pirfenidone: results of a prospective, open-label Phase II study. Am J Respir Crit Care Med 1999;159:1061-9

74. Azuma A, Nukiwa T, Tsuboi E, et al. Double-blind, placebo-controlled trial of pirfenidone in patients with idiopathic pulmonary fibrosis. Am J Respir Crit Care Med 2005;171:1040-7

75. Taniguchi H, Ebina M, Kondoh Y, et al. Pirfenidone in idiopathic pulmonary fibrosis. Eur Respir J 2010;35:821-9

76. InterMune, Inc. Sponsor's Briefing Document for the FDA Pulmonary-Allergy Drugs Advisory Committee Meeting (NDA 22-535); 9 March 2010

77. Clark JG, Dedon TF, Wayner EA, Carter WG. Effects of interferon-gamma on expression of cell surface receptors for collagen and deposition of newly synthesized collagen by cultured human lung fibroblasts. J Clin Invest 1989;83:1505-11

78. Narayanan AS, Whithey J, Souza A, Raghu G. Effect of gamma-interferon on collagen synthesis by normal and fibrotic human lung fibroblasts. Chest 1992;101:1326-31

79. Okada T, Sugie I, Aisaka K. Effects of gamma-interferon on collagen and histamine content in bleomycin-induced lung fibrosis in rats.

Lymphokine Cytokine Res 1993;12:87-91

80. Gurujeyalakshmi G, Giri SN. Molecular mechanisms of antifibrotic effect of interferon gamma in bleomycin-mouse model of lung fibrosis: downregulation of TGF-beta and procollagen I and III gene expression. Exp Lung Res 1995;21:791-808

81. Ziesche R, Hofbauer E, Wittmann K, et al. A preliminary study of long-term treatment with interferon gamma- $1 \mathrm{~b}$ and low-dose prednisolone in patients with idiopathic pulmonary fibrosis. N Engl J Med 1999;341:1264-9

82. Raghu G, Brown KK, Bradford WZ, et al. A placebo-controlled trial of interferon gamma- $1 \mathrm{~b}$ in patients with idiopathic pulmonary fibrosis. N Engl J Med 2004;350:125-33

83. King TE Jr, Albera C, Bradford WZ, et al. Effect of interferon gamma-1b on survival in patients with idiopathic pulmonary fibrosis (INSPIRE): a multicentre, randomised, placebo-controlled trial. Lancet 2009;374:222-8

84. Day E, Waters B, Spiegel K, et al. Inhibition of collagen-induced discoidin domain receptor 1 and 2 activation by imatinib, nilotinib and dasatinib. Eur J Pharmacol 2008;599:44-53

85. Buchdunger E, O’Reilly T, Wood J. Pharmacology of imatinib (STI571). Eur J Cancer 2002; 38:(Suppl 5):S28-36

86. Aono $\mathrm{Y}$, Nishioka $\mathrm{Y}$, Inayama $\mathrm{M}$, et al. Imatinib as a novel antifibrotic agent in bleomycin-induced pulmonary fibrosis in mice. Am J Respir Crit Care Med 2005;171:1279-85

87. Daniels CE, Lasky JA, Limper AH, et al. Imatinib treatment for idiopathic pulmonary fibrosis: randomized placebo-controlled trial results. Am J Respir Crit Care Med 2010;181:604-10

88. Shi-Wen X, Denton CP, Dashwood MR, et al. Fibroblast matrix gene expression and connective tissue remodeling: role of endothelin-1. J Invest Dermatol 2001;116:417-25

89. Park SH, Saleh D, Giaid A, Michel RP. Increased endothelin-1 in bleomycin-induced pulmonary fibrosis and the effect of an endothelin receptor antagonist. Am J Respir Crit Care Med. 1997; 156:600-8

90. King TE Jr, Behr J, Brown KK, et al. BUILD-1: a randomized placebo-controlled trial of bosentan in 
idiopathic pulmonary fibrosis. Am J Respir Crit Care Med. 2008;177:75-81

91. Raghu G, Brown KK, Costabel U, et al. Treatment of idiopathic pulmonary fibrosis with etanercept: an exploratory, placebo-controlled trial. Am J Respir Crit Care Med 2008;178:948-55

92. Grimminger F, Schermuly RT, Ghofrani HA. Targeting non-malignant disorders with tyrosine kinase inhibitors. Nat Rev Drug Discov 2010;9:956-70

93. Allen JT, Spiteri MA. Growth factors in idiopathic pulmonary fibrosis: relative roles. Respir Res 2002;3:13

94. Chaudhary NI, Roth GJ, Hilberg F, et al. Inhibition of PDGF, VEGF and FGF signalling attenuates fibrosis. Eur Respir J 2007;29:976-85

95. Moeller A, Ask K, Warburton D, et al The bleomycin animal model: a useful tool to investigate treatment options for idiopathic pulmonary fibrosis? Int J Biochem Cell Biol 2008;40:362-82

96. Moore BB, Hogaboam CM. Murine models of pulmonary fibrosis. Am J Physiol Lung Cell Mol Physiol 2008;294:L152-60

97. Hecker L, Vittal R, Jones $\mathrm{T}$, et al. NADPH oxidase- 4 mediates myofibroblast activation and fibrogenic responses to lung injury. Nat Med 2009; 15:1077-81

98. Pache JC, Carnesecchi S, Deffert C, et al. NOX-4 is expressed in thickened pulmonary arteries in idiopathic pulmonary fibrosis. Nat Med 2011;17:31-2; author reply 2-3

99. Amara N, Goven D, Prost F, et al. NOX4/NADPH oxidase expression is increased in pulmonary fibroblasts from patients with idiopathic pulmonary fibrosis and mediates TGFbeta1-induced fibroblast differentiation into myofibroblasts. Thorax 2010;65:733-8

100. Laleu B, Gaggini F, Orchard M, et al. First in class, potent, and orally bioavailable NADPH oxidase isoform 4 (Nox4) inhibitors for the treatment of idiopathic pulmonary fibrosis. J Med Chem 2010;53:7715-30

101. Hinz B. Tissue stiffness, latent TGF-beta1 activation, and mechanical signal transduction: implications for the pathogenesis and treatment of fibrosis. Curr Rheumatol Rep 2009;11:120-6

102. Kagan HM, Li WD. Lysyl oxidase: properties, specificity, and biological roles inside and outside of the cell.

J Cell Biochem 2003;88:660-72

103. Barry-Hamilton V, Spangler R, Marshall D, et al. Allosteric inhibition of lysyl oxidase-like- 2 impedes the development of a pathologic microenvironment. Nat Med 2010;16:1009-17

104. Kubo H, Nakayama K, Yanai M, et al. Anticoagulant therapy for idiopathic pulmonary fibrosis. Chest 2005; 128:1475-82

105. Imokawa $S$, Sato $A$, Hayakawa $H$, et al. Tissue factor expression and fibrin deposition in the lungs of patients with idiopathic pulmonary fibrosis and systemic sclerosis. Am J Respir Crit Care Med 1997;156:631-6

106. Hernandez-Rodriguez NA, Cambrey AD, Harrison NK, et al. Role of thrombin in pulmonary fibrosis. Lancet 1995;346:1071-3

107. Howell DC, Goldsack NR, Marshall RP, et al. Direct thrombin inhibition reduces lung collagen, accumulation, and connective tissue growth factor mRNA levels in bleomycin-induced pulmonary fibrosis. Am J Pathol 2001;159:1383-95

108. Gunther A, Lubke N, Ermert M, et al. Prevention of bleomycin-induced lung fibrosis by aerosolization of heparin or urokinase in rabbits. Am J Respir Crit Care Med 2003;168:1358-65

109. Blanc-Brude OP, Chambers RC, Leoni $\mathrm{P}$, et al. Factor $\mathrm{Xa}$ is a fibroblast mitogen via binding to effector-cell protease receptor- 1 and autocrine release of PDGF. Am J Physiol Cell Physiol 2001;281:C681-9

110. Chambers RC, Leoni P, Blanc-Brude OP, et al. Thrombin is a potent inducer of connective tissue growth factor production via proteolytic activation of protease-activated receptor-1. J Biol Chem 2000;275:35584-91

111. Jenkins RG, Su X, Su G, et al. Ligation of protease-activated receptor 1 enhances alpha(v)beta(6) integrin-dependent TGF-beta activation and promotes acute lung injury. J Clin Invest 2006;116:1606-14

112. Howell DCJ, Johns RH, Lasky JA, et al. Absence of proteinase-activated receptor-1 signaling affords protection from bleomycin-induced lung inflammation and fibrosis. Am J Pathol 2005;166:1353-65

113. Becker RC, Moliterno DJ, Jennings LK, et al. Safety and tolerability of $\mathrm{SCH}$ 530348 in patients undergoing non-urgent percutaneous coronary intervention: a randomised, double-blind, placebo-controlled Phase II study. Lancet 2009;373:919-28

114. Pilling D, Buckley CD, Salmon M, Gomer RH. Inhibition of fibrocyte differentiation by serum amyloid P. J Immunol 2003;171:5537-46

115. Pilling D, Roife D, Wang M, et al. Reduction of bleomycin-induced pulmonary fibrosis by serum amyloid P. J Immunol 2007;179:4035-44

116. Wang J, Jiao H, Stewart TL, et al. Improvement in postburn hypertrophic scar after treatment with IFN-alpha2b is associated with decreased fibrocytes. J Interferon Cytokine Res 2007;27:921-30

117. Katebi M, Fernandez P, Chan ES, Cronstein BN. Adenosine A2A receptor blockade or deletion diminishes fibrocyte accumulation in the skin in a murine model of scleroderma, bleomycin-induced fibrosis. Inflammation 2008;31:299-303

118. Vannella KM, McMillan TR, Charbeneau RP, et al. Cysteinyl leukotrienes are autocrine and paracrine regulators of fibrocyte function. J Immunol 2007;179:7883-90

119. Phillips RJ, Burdick MD, Hong K, et al. Circulating fibrocytes traffic to the lungs in response to CXCL12 and mediate fibrosis. J Clin Invest 2004;114:438-46

120. Wang Z, Li Y, Ahmad A, et al. Targeting miRNAs involved in cancer stem cell and EMT regulation: An emerging concept in overcoming drug resistance. Drug Resist Updat 2010;13:109-18

121. Nagai T, Arao T, Furuta K, et al. Sorafenib inhibits the hepatocyte growth factor-mediated epithelial mesenchymal transition in hepatocellular carcinoma. Mol Cancer Ther 2011;10:169-77 
122. Farkas L, Farkas D, Gauldie J, et al Transient overexpression of gremlin results in epithelial activation and reversible fibrosis in rat lungs. Am J Respir Cell Mol Biol 2010

123. Becerril C, Pardo A, Montano M, et al. Acidic fibroblast growth factor induces an antifibrogenic phenotype in human lung fibroblasts. Am J Respir Cell Mol Biol 1999;20:1020-7

124. Ramos C, Montano M, Becerril C, et al. Acidic fibroblast growth factor decreases alpha-smooth muscle actin expression and induces apoptosis in human normal lung fibroblasts. Am J Physiol Lung Cell Mol Physiol 2006;291:L871-9

125. Ramos C, Becerril C, Montano M, et al. FGF-1 reverts epithelial-mesenchymal transition induced by TGF-\{beta\} 1 through MAPK/ERK kinase pathway. Am J Physiol Lung Cell Mol Physiol 2010;299:L222-31

126. Watanabe M, Ebina M, Orson FM, et al. Hepatocyte growth factor gene transfer to alveolar septa for effective suppression of lung fibrosis. Mol Ther 2005;12:58-67

127. Guo J, Yi ES, Havill AM, et al. Intravenous keratinocyte growth factor protects against experimental pulmonary injury. Am J Physiol 1998;275:L800-5

128. Aguilar S, Scotton CJ, McNulty K, et al. Bone marrow stem cells expressing keratinocyte growth factor via an inducible lentivirus protects against bleomycin-induced pulmonary fibrosis. PLoS One 2009; 4:e8013

129. Sakamoto S, Yazawa T, Baba Y, et al. Keratinocyte growth factor gene transduction ameliorates pulmonary fibrosis induced by bleomycin in mice. Am J Respir Cell Mol Biol 2010

130. Spielberger R, Stiff P, Bensinger W, et al. Palifermin for oral mucositis after intensive therapy for hematologic cancers. N Engl J Med 2004;351:2590-8

131. Rubenstein EB, Peterson DE, Schubert M, et al. Clinical practice guidelines for the prevention and treatment of cancer therapy-induced oral and gastrointestinal mucositis. Cancer 2004;100:2026-46

132. Kobbe G, Bruns I, Schroeder T, et al. A 3-day short course of palifermin before HDT reduces toxicity and need for supportive care after autologous blood stem-cell transplantation in patients with multiple myeloma. Ann Oncol 2010;21:1898-904

133. Henderson WR Jr, Chi EY, Ye X, et al. Inhibition of Wnt/beta-catenin/CREB binding protein (CBP) signaling reverses pulmonary fibrosis. Proc Natl Acad Sci USA 2010;107:14309-14

134. Crosby LM, Waters CM. Epithelial repair mechanisms in the lung. Am J Physiol Lung Cell Mol Physiol 2010;298:L715-31

135. Weiss DJ, Kolls JK, Ortiz LA, et al. Stem cells and cell therapies in lung biology and lung diseases. Proc Am Thorac Soc 2008;5:637-67

136. De Miguel MP, Fuentes-Julian S, Alcaina Y. Pluripotent stem cells: origin, maintenance and induction. Stem Cell Rev 2010;6:633-49

137. Wang D, Haviland DL, Burns AR, et al. A pure population of lung alveolar epithelial type II cells derived from human embryonic stem cells. Proc Natl Acad Sci USA 2007;104:44499-54

138. Wang D, Morales JE, Calame DG, et al. Transplantation of human embryonic stem cell-derived alveolar epithelial type II cells abrogates acute lung injury in mice. Mol Ther 2010;18:625-34

139. Wernig M, Meissner A, Foreman R, et al. In vitro reprogramming of fibroblasts into a pluripotent ES-cell-like state. Nature 2007;448:318-24

140. Okita K, Ichisaka T, Yamanaka S. Generation of germline-competent induced pluripotent stem cells. Nature 2007;448:313-17

141. Pfannkuche K, Hannes T, Khalil M, et al. Induced pluripotent stem cells: a new approach for physiological research. Cell Physiol Biochem 2010;26:105-24

142. Somers A, Jean JC, Sommer CA, et al. Generation of transgene-free lung disease-specific human induced pluripotent stem cells using a single excisable lentiviral stem cell cassette. Stem Cells 2010;28:1728-40

143. Ortiz LA, Gambelli F, McBride C, et al. Mesenchymal stem cell engraftment in lung is enhanced in response to bleomycin exposure and ameliorates its fibrotic effects. Proc Natl Acad Sci USA 2003;100:8407-11
144. Rojas M, Xu J, Woods CR, et al. Bone marrow-derived mesenchymal stem cells in repair of the injured lung. Am J Respir Cell Mol Biol 2005;33:145-52

145. Moodley Y, Atienza D, Manuelpillai U, et al. Human umbilical cord mesenchymal stem cells reduce fibrosis of bleomycin-induced lung injury. Am J Pathol 2009;175:303-13

146. Lee SH, Jang AS, Kim YE, et al. Modulation of cytokine and nitric oxide by mesenchymal stem cell transfer in lung injury/fibrosis. Respir Res 2010;11:16

147. Kotton DN, Fabian AJ, Mulligan RC. Failure of bone marrow to reconstitute lung epithelium. Am J Respir Cell Mol Biol 2005;33:328-34

148. Chang JC, Summer R, Sun X, et al. Evidence that bone marrow cells do not contribute to the alveolar epithelium. Am J Respir Cell Mol Biol 2005;33:335-42

149. Charbord P. Bone marrow mesenchymal stem cells: historical overview and concepts. Hum Gene Ther 2010;21:1045-56

150. Riggi N, Suva ML, De Vito C, et al. EWS-FLI-1 modulates miRNA145 and SOX2 expression to initiate mesenchymal stem cell reprogramming toward Ewing sarcoma cancer stem cells. Genes Dev 2010;24:916-32

151. Prockop DJ, Kota DJ, Bazhanov N, Reger RL. Evolving paradigms for repair of tissues by adult stem/progenitor cells (MSCs). J Cell Mol Med. 2010;14:2190-9

152. Price AP, England KA, Matson AM, et al. Development of a decellularized lung bioreactor system for bioengineering the lung: the matrix reloaded. Tissue Eng Part A 2010;16:2581-91

153. Petersen TH, Calle EA, Zhao L, et al. Tissue-engineered lungs for in vivo implantation. Science 2010;329:538-41

154. Pandit KV, Corcoran D, Yousef H, et al. Inhibition and role of let- $7 \mathrm{~d}$ in idiopathic pulmonary fibrosis. Am J Respir Crit Care Med 2010;182:220-9

155. Liu G, Friggeri A, Yang Y, et al. miR-21 mediates fibrogenic activation of pulmonary fibroblasts and lung fibrosis. J Exp Med 2010;207:1589-97 


\title{
Emerging drugs for idiopathic pulmonary fibrosis
}

156. Cushing L, Kuang PP, Qian J, et al. MIR-29 is a Major regulator of genes associated with pulmonary fibrosis. Am J Respir Cell Mol Biol 2010

157. Bader AG, Brown D, Winkler M. The promise of microRNA replacement therapy. Cancer Res 2010;70:7027-30

158. Kumar MS, Erkeland SJ, Pester RE, et al. Suppression of non-small cell lung tumor development by the let-7 microRNA family. Proc Natl Acad Sci USA 2008;105:3903-8

159. Wiggins JF, Ruffino L, Kelnar K, et al. Development of a lung cancer therapeutic based on the tumor suppressor microRNA-34. Cancer Res 2010;70:5923-30

\author{
Affiliation \\ Moisés Selman ${ }^{\dagger 1}$, Annie Pardo ${ }^{2}$, \\ Luca Richeldi ${ }^{3} \&$ Stefania Cerri ${ }^{3}$ \\ ${ }^{\dagger}$ Author for correspondence \\ ${ }^{1}$ Instituto Nacional de Enfermedades \\ Respiratorias, \\ Tlalpan 4502; CP 14080, \\ México DF, México \\ Tel: +52 555487 1771; Fax: +52 555665 4623; \\ E-mail: mselmanl@yahoo.com.mx \\ ${ }^{2}$ Facultad de Ciencias, \\ Universidad Nacional Autónoma de México, \\ Mexico \\ ${ }^{3}$ Center for Rare Lung Disease, \\ University of Modena and Reggio Emilia, \\ Modena, Italy
}

